\title{
Mid-infrared properties of nearby low-luminosity AGN at high angular resolution $\star, \star \star$
}

\author{
D. Asmus ${ }^{1,2}$, P. Gandhi ${ }^{3}$, A. Smette ${ }^{1}$, S. F. Hönig ${ }^{4}$, and W. J. Duschl ${ }^{2,5}$
}

\author{
1 European Southern Observatory, Casilla 19001, Santiago 19, Chile \\ e-mail: asmus@astrophysik.uni-kiel.de \\ 2 Institut für Theoretische Physik und Astrophysik, Christian-Albrechts-Universität zu Kiel, Leibnizstr. 15, 24098 Kiel, Germany \\ ${ }^{3}$ Institute of Space and Astronautical Science (ISAS), Japan Aerospace Exploration Agency, 3-1-1 Yoshinodai, chuo-ku, Sagamihara, \\ Kanagawa 252-5210, Japan \\ 4 Department of Physics, University of California in Santa Barbara, Broida Hall, Santa Barbara, CA 93106-9530, USA \\ 5 Steward Observatory, The University of Arizona, 933 N. Cherry Ave, Tucson, AZ 85721, USA \\ Received 10 February 2011 / Accepted 19 September 2011
}

\section{ABSTRACT}

\begin{abstract}
We present high spatial resolution mid-infrared (MIR) $12 \mu \mathrm{m}$ continuum imaging of low-luminosity active galactic nuclei (LLAGN) obtained with VLT/VISIR. Our goal is to determine whether the nuclear MIR emission of LLAGN is consistent with the existence of a dusty obscuring torus, the key component of the unification model for AGN. Based on available hard X-ray luminosities and the previously known tight correlation between the hard X-ray and $12 \mu \mathrm{m}$ luminosities, we selected a sample of 17 nearby LLAGN without available VISIR $N$-band photometry. Combined with archival VISIR data of 9 additional LLAGN with available X-ray measurements, the dataset represents the bulk of southern LLAGN currently detectable from the ground in the MIR. Of the 17 observed LLAGN, 7 are detected, while upper limits are derived for the 10 non-detections. This increases the total number of AGN detected with VLT/VISIR to more than 50. All detections except NGC 3125 appear point-like on a spatial scale of $\sim 0.35^{\prime \prime}$. The detections do not significantly deviate from the known MIR-X-ray correlation but exceed it by a factor of $\sim 10$ down to luminosities $<10^{41} \mathrm{erg} / \mathrm{s}$ with a narrow scatter $(\sigma=0.35 \mathrm{dex}$, Spearman rank $\rho=0.92)$. The latter is dominated by the uncertainties in the X-ray luminosity. Interestingly, a similar correlation with a comparable slope but with a normalization differing by $\sim 2.6$ orders of magnitude has been found for local starburst galaxies. In addition, we compared the VISIR data with lower spatial resolution data from Spitzer/IRS and IRAS. By using a scaled starburst template spectral energy distribution and the polycyclic aromatic hydrocarbon (PAH) $11.3 \mu \mathrm{m}$ emission line, we were able to restrict the maximum nuclear star-formation contamination of the VISIR photometry to $\$ 30 \%$ for $75 \%$ of the LLAGN. Exceptions are NGC 1097 and NGC 1566, which may possess unresolved strong PAH emission. Furthermore, the MIR$\mathrm{X}$-ray luminosity ratio is unchanged over more than 4 orders of magnitude in accretion rate within the uncertainties. These results are consistent with the existence of the dusty torus in all observed LLAGN, although a jet or accretion disk as origin of the MIR emission cannot be excluded. Finally, because the MIR-X-ray correlation holds for all LLAGN and Seyferts, this is a very useful empirical tool for converting between the MIR and X-ray powers of these nuclei.
\end{abstract}

Key words. accretion, accretion disks - galaxies: active - infrared: galaxies - galaxies: nuclei - galaxies: Seyfert - X-rays: galaxies

\section{Introduction}

Low-luminosity active galactic nuclei (LLAGN; $L_{2-10 \mathrm{keV}} \lesssim$ $10^{42} \mathrm{erg} / \mathrm{s}$ ) have drawn increasing attention in recent years for various reasons: it has been realized that this population is not only the most common of all active galaxies (AGN) - about twothirds of all AGN are LLAGN (see Ho 2008) - but they may also represent AGN in the initial or final stages of their active phases. The underlying idea is that every galaxy harbors a black hole $(\mathrm{BH})$ in its center that grows and evolves to a supermassive $\mathrm{BH}$ during many phases of nuclear activity. The key components describing AGN in the so-called unification scheme (Antonucci 1993) such as the accretion disk and the dusty obscuring torus very likely vanish during non-active phases and are built up again when new material reaches the galactic nucleus through any kind of larger-scale disturbance. But it is unknown how the processes of formation and evolution of both are connected. This is partly because the physical nature and morphology of the disk

\footnotetext{
* Based the ESO observing programs 083.B-0536 and 086.B-0349.

$\star \star$ Appendices are available in electronic form at

http://www. aanda.org
}

and torus is still not exactly known. However, high spatial resolution mid-infrared (MIR) observations made progress in the modeling of the torus possible. They favor a clumpy distribution of optically thick clouds with a small filling factor (e.g., Nenkova et al. 2002; Dullemond \& van Bemmel 2005; Hönig et al. 2006; Schartmann et al. 2008; Nenkova et al. 2008; Hönig \& Kishimoto 2010). Apart from the fact that the mechanism that maintains the geometrical thickness of the torus is unknown, there are various theoretical works predicting its disappearance below certain bolometric luminosities or accretion rates (Elitzur \& Shlosman 2006; Hönig \& Beckert 2007). But observational proof of this disappearance is still lacking.

The putative dusty torus of AGN is best observed in the MIR, where the thermal emission from the warm dust is strongest. Ground-based MIR instruments like VLT/VISIR (Lagage et al. 2004) are best suited to perform these observations because of their high spatial resolution. And an increasing number of AGN studies demonstrate their power to isolate the AGN from surrounding starbursts (e.g., Gorjian et al. 2004; Galliano et al. 2005; Haas et al. 2007; Siebenmorgen et al. 2008; Ramos Almeida et al. 2009). Furthermore, mid-infrared interferometric 
observations were able to resolve the dusty torus in nearby AGN and showed that it is only a few parsec in size (e.g., Jaffe et al. 2004; Raban et al. 2009; Tristram et al. 2009; Burtscher et al. 2009) and scales by the square root of the AGN luminosity (Tristram \& Schartmann 2011).

On the other hand, past low-resolution MIR observations led to the discovery of a correlation between the MIR and X-ray luminosities (Krabbe et al. 2001; Lutz et al. 2004). Here, the observed nuclear MIR emission is believed to represent the reprocessed UV/X-ray radiation from the dusty clouds inside the torus. This conclusion is strongly supported by fitting of highspatial resolution spectrophotometry with clumpy torus models (Hönig et al. 2010; Alonso-Herrero et al. 2011).

Then, recently gathered VISIR photometry enabled the best estimate of AGN emission around 12 micron, free of stellar contamination, resulting in a strong correlation with the intrinsic X-ray 2-10 keV luminosity (Horst et al. 2006, 2008, 2009; Gandhi et al. 2009), which is well-described by a power-law $\left(L_{\mathrm{MIR}} \propto L_{\mathrm{X}}^{b}\right)$ with a slope $b \sim 1$. Similar results have also been found with other ground-based high spatial resolution MIR instruments (e.g., Levenson et al. 2009). The luminosity correlation for the different AGN types is indistinguishable within the measured precision, and it can be used, e.g., to assess the bolometric IR luminosity of AGN when no high-resolution IR data are available (e.g., Mullaney et al. 2011).

However, the lowest luminosity objects of the observed samples (in, e.g., Gandhi et al. 2009, hereafter G+09), in particular the three low-ionization nuclear emission-line regions (LINERs), show a tendency to deviate from this correlation: all objects with an X-ray luminosity $L_{2-10 \mathrm{keV}}<10^{42} \mathrm{erg} / \mathrm{s}$ exhibit a MIR excess (or an X-ray deficit) of $\sim 0.3 \mathrm{dex}$. The total number of included LLAGN was too small to give statistical significant evidence for a real deviation. While this deviation could indicate a change in the physical structure of the AGN at low luminosities, one first has to exclude other causes such as, e.g., significant MIR emission from dust heated by circum-nuclear starformation regions. In particular at low luminosities, the latter can be of comparable MIR brightness with respect to the AGN or even brighter. Furthermore, the proposed collapse of the dusty torus (see above) would lead to a deficit of MIR emission, opposite to the indicated deviation. Instead, a separate AGN component would have to explain the MIR excess (or X-ray deficit) at low luminosities, if real. One candidate would be the jet, which is known to emit copious amounts of synchrotron emission over the whole observable wavelength range. Particularly in LINERs (a sub-class of LLAGN) the jet is assumed to be much more powerful than in Seyferts, which explains the relative radio-brightness of these optically faint objects. For example, indications of a significant jet contribution to the MIR emission of LLAGN was found in Hardcastle et al. (2009). Indeed the observed MIR emission for NGC 4486, a well-known LINER, can be explained only with synchrotron emission of different jet components (Perlman et al. 2007).

However, the inner accretion disk might also emit MIR synchrotron emission, when in the so-called radiatively inefficient accretion flow (RIAF) or advection-dominated accretion flow (ADAF) mode. The latter was theoretically predicted by Narayan \& Yi (1994) to occur at low accretion rates: the standard geometrically thin, optically thick disk (Shakura \& Sunyaev 1973) transforms into a geometrically thick, optically thin accretion disk in the inner part. In addition, the thin accretion disk may still exist in the outer parts as a so-called truncated disk emitting thermal MIR radiation. Later, observations indeed gave evidence for this accretion mode in LLAGN, and thus it is favored for these sources (see Ho 2008; Yuan 2007, for an overview). Of course, a change in the accretion mode could also invoke a change in the UV or X-ray emission properties, which could eventually lead by itself to an observed X-ray deficit (or MIR excess) compared to Seyferts accreting in a different mode.

To summarize, circum-nuclear star-formation, the putative torus, a jet or the accretion disk can all significantly contribute to the observed MIR emission of LLAGN. By characterizing the MIR spectral energy distribution, it might be possible to distinguish between these different possible components, or at least constrain their relative strength - and thus test the underlying models for LLAGN. We present the first step by investigating whether the apparent deviation from the luminosity correlation of $\mathrm{G}+09$ still holds for a larger sample of LLAGN, and by finding possible explanations for any deviation. To this aim, we present new VISIR imaging data of a sample of 17 LLAGN and compare it to the previous higher luminosity AGN sample from $\mathrm{G}+09$.

The structure of this paper is as follows: first, the sample selection and properties are described in Sect. 2. The following Sect. 3 details the VISIR observations, MIR data reduction and photometric measurements. The obtained results are presented in Sect. 4. And combined with additional objects from the literature, the MIR-X-ray correlation for LLAGN is investigated. Comparisons to Spitzer/IRS and IRAS are presented and discussed in Sect. 5, as well as possible star-formation contamination. In addition, a possible dependence of the MIR-X-ray luminosity ratio on the accretion rate is discussed in Sect. 5.4. The paper ends with a summary and conclusions (Sect. 6).

\section{Properties of the LLAGN sample}

The sample of LLAGN was chosen with the goal of testing the previously found MIR excess/X-ray deficit. Thus, the sample is not suited to detect objects significantly above the correlation (i.e. showing a MIR deficit). We specifically selected 17 LLAGN according to the following main criteria: i) they are visible from Paranal, ii) they have a nuclear X-ray detection in the $2-10 \mathrm{keV}$ band with a corrected $L_{2-10 \mathrm{keV}} \lesssim 10^{42} \mathrm{erg} / \mathrm{s}$; iii) they are classified as LINER or Seyfert; and iv) they show an expected flux $f_{12 \mu \mathrm{m}} \geq 10 \mathrm{mJy}$ assuming that the objects follow the MIR$\mathrm{X}$-ray correlation as found by Horst et al. (2008) (nine objects) or that they follow the trend of MIR excess (or X-ray deficit) seen for the three LINERS in the same work (nine objects). A flux of $10 \mathrm{mJy}$ is roughly the lowest unresolved $12 \mu \mathrm{m}$ flux that VLT/VISIR can detect in a narrow-band filter in a reasonable amount of execution time $(\sim 1 \mathrm{~h})$ under average conditions. In addition, there are objects for which the selection criteria apply, but which have sufficient VISIR $N$-band imaging data available in the literature (e.g., Centaurus A, M 87). These objects will be included in the latter part of this analysis. Thus, the total sample comprises 26 LLAGN with 11 LINERs, 2 type 1 Seyferts and 13 type 2 Seyfert. In addition, NGC 1404, which is classified as a narrow-emission-line galaxy (NELG), was included in this sample because it was expected to be bright enough in the MIR. We will distinguish only the following main classes: type $1(1.0-1.5)$, type $2(1.8-2.0$ and $1 \mathrm{~h}, 1 \mathrm{li})$ and LINERs. The AGN classification of Véron-Cetty \& Véron (2010) have been used in all cases where available (see Table 2). NGC 3125, NGC 5363, NGC 5813, and NGC 7626 are not in this catalog, but have been classified as LINERs by Carrillo et al. (1999) and Ho et al. (1995). Note that some objects have multiple different classifications (as either Seyfert or LINER) in the literature (e.g., NGC 1097 or NGC 7213). All these classifications are defined 
by optical properties (emission line ratios), which can be heavily affected by extinction. Furthermore, a significant number of AGN might even be completely obscured in the optical and thus remain unidentified as active (e.g., Goulding \& Alexander 2009).

Additional notes on individual LLAGN included in this work, in particular concerning their X-ray properties, can be found in Appendix B.

\subsection{Distances}

Because the selected LLAGN are very nearby in a cosmological sense $(\sim 20 \mathrm{Mpc})$, the motion caused by local gravity potentials can dominate the Hubble flow. Therefore it can be necessary for most nearby galaxies to use redshift-independent distance measurement methods. We used three different distance collections: a) for comparison the redshift-based luminosity distances $D_{\mathrm{L}}$ from NED using redshifts corrected for the Earth motion relative to the cosmic microwave background reference frame with $H_{0}=73, \Omega_{\mathrm{m}}=0.27$, and $\left.\Omega_{\mathrm{vac}}=0.73 ; \mathrm{b}\right)$ the distances given by Tully (1988) employing the Tully-Fisher (TF) relation, and c) distances of various methods with preference given to the most precise and recent measurements, with the highest priority given to direct Cepheid-based distance measurements and next using the surface brightness fluctuation method (see Jacoby et al. 1992 , for a review and comparison). If not available, the values based on the TF method or $D_{\mathrm{L}}$ were used. Note that there is no Tully-Fisher distance available for NGC 1667, NGC 3125 and NGC 7626. References for the individual sources are listed in Table 2. However, unless noted otherwise distance set c) will be used.

\subsection{X-ray luminosities}

The X-ray luminosities were selected from the literature and were corrected for the distances adopted in each case. We preferred the most recent observations with X-ray telescopes that had a high angular resolution, a large effective area, broad band energy coverage or the best combination of these. The values used are listed in Table 2 and represent in all cases the intrinsic luminosities including absorption. Therefore, they can of course depend strongly on the spectral models used. This mostly affects Compton-thick (CT, $N_{\mathrm{H}}>1.5 \times 10^{24} \mathrm{~cm}^{-2}$ ) objects, and a number of LLAGN turned out to be such CT candidates. Their $\mathrm{X}$-ray spectra are often dominated by scattered or extended emission and consequently appear to be unabsorbed with a much lower luminosity than predicted by CT obscuration. Depending on the model assumed, this leads to intrinsic $2-10 \mathrm{keV}$ luminosity estimates differing by several orders of magnitude (e.g., for NGC 7743).

To identify the "true" nature of the Compton-thick candidates, it is useful to compare the estimated X-ray luminosity to other intrinsic indicators, such as luminosities of the forbidden emission lines [OIII] $\lambda 5007 \AA$ and [OIV] $\lambda 25.89 \mu \mathrm{m}$. In Panessa et al. (2006) a correlation between the $2-10 \mathrm{keV}$ and [OIII] luminosities has been found, which can be used to predict the intrinsic X-ray luminosity for the Compton-thick candidates. There is also evidence for significant absorption of [OIII] in the narrowline region (NLR; Haas et al. 2005). But the [OIV] is almost unabsorbed, which makes it a powerful tool (Meléndez et al. 2008). Diamond-Stanic et al. (2009) present an extended sample of local Seyferts with measured [OIV] and observed X-ray fluxes, whose luminosities correlate well for the 27 unabsorbed sources (Spearman rank $\rho=0.84$ with $\log p=-7.3$ ). A bisector fit (Isobe et al. 1990) to these objects yields

$\log \left(\frac{L_{2-10 \mathrm{keV}}}{\mathrm{erg} \mathrm{s}^{-1}}\right)=-0.44 \pm 3.46+(1.06 \pm 0.09)\left(\frac{L_{[\mathrm{OIV}]}}{\mathrm{erg} \mathrm{s}^{-1}}\right)$.

Both luminosity correlations, X-ray-[OIII] and X-ray-[OIV], are used to predict the intrinsic X-ray luminosities of the CT candidates, and thus to distinguish between the Compton-thin or thick scenario.

In addition, it is well known that AGN can show huge X-ray variation, either intrinsic or caused by a change of obscuration/absorption in the innermost region (e.g., Murphy et al. 2007; Risaliti et al. 2007). To account for this, we assumed an error of factor $2(0.3 \mathrm{dex})$ in all cases where variation is evident or only one high spatial resolution X-ray observation is available, while we applied 0.6 dex for CT candidates. In particular, it is important to note that X-ray luminosities of the following objects have been significantly revised since the sample was initially selected: for NGC 5813 only ROSAT data were available, inferring a much higher X-ray luminosity ( $\log L_{2-10 \mathrm{keV}}=42.1$, Schwope et al. 2000) than later found by Chandra $\left(\log L_{2-10} \mathrm{keV}=38.77\right)$, similarly, for NGC 7590 the $A S C A$ data yielded $\log L_{2-10 \mathrm{keV}}=40.78$ (Bassani et al. 1999) and later XMM-Newton $\log L_{2-10 \mathrm{keV}}=$ 39.70 (Shu et al. 2010a). This led to a severe overestimation of the MIR flux and thus an underestimation of the VISIR exposure times for these objects.

\section{Observations and data reduction}

For each target we chose a set of two filters, such that the bandpass of one contained only continuum emission around $12 \mu \mathrm{m}$, while the other contained the polycyclic aromatic hydrocarbon (PAH) emission feature at $11.3 \mu \mathrm{m}$. Because all objects are at very low redshifts $(\langle z\rangle=0.006)$, the NeIIref1 $\left(\lambda_{\mathrm{c}}=12.27 \mu \mathrm{m}\right.$, half-band width $=0.18 \mu \mathrm{m})$ and PAH2 $\left(\lambda_{\mathrm{c}}=11.25 \mu \mathrm{m}\right.$, halfband width $=0.59 \mu \mathrm{m}$ ) filters were used in all cases.

The VLT/VISIR observations were carried out in service mode between April and September 2009 (ESO period P83). Most observations fulfilled the required observing conditions (airmass $\leq 1.3$, optical seeing $\leq 0$ ' 8 , MIR photometric sensitivity within $20 \%$ of expected values). An exception is IC 1459 , which was observed in conditions that resulted in a much poorer sensitivity. Note that NGC 676 was observed again in P86 with longer exposure times and the more sensitive PAH2ref2 filter $\left(\lambda_{\mathrm{c}}=11.88 \mu \mathrm{m}\right.$, half-band width $\left.=0.37 \mu \mathrm{m}\right)$. These data will be used here instead of the NeIIref1 data from P83.

Standard chopping and nodding observing mode with a chop throw of $8^{\prime \prime}$ on the small field-of-view $(19,2 \times 19$ !' 2 ; 0 .'075/pixel) was performed. Therefore, all four beams fell on the detector - for science targets the two positive ones top of each other (parallel mode), ensuring the highest possible signal-to-noise $(S / N)$ for this observing mode. After consulting Spitzer/IRAC $8 \mu \mathrm{m}$ images (or 2MASS if the former was not available), we chose the chopping throw and angle for each individual object such that overlap of the nucleus with non-nuclear emission regions was avoided. The flux level of the diffuse host emission at a distance of $8^{\prime \prime}$ drops to less than $10 \%$ compared to the nucleus in $80 \%$ of the cases. Accordingly, possible contamination through chopping induced overlaps is estimated to be $<10 \%$. The most severe exception is NGC 7590, which shows very complex extended MIR morphologies in IRAC. Therefore, it was observed with the generic nodding template using offset of $30^{\prime \prime}$, but it remained undetected with VISIR. For each science 
Table 1. Observational parameters and reduction results for the P83 sample.

\begin{tabular}{|c|c|c|c|c|c|c|c|}
\hline \multirow[t]{2}{*}{ Object } & \multirow{2}{*}{$\begin{array}{c}\text { Obs. date } \\
\text { (YY-MM-DD) }\end{array}$} & \multirow[t]{2}{*}{ Filter } & \multirow{2}{*}{$\begin{array}{c}\lambda_{\text {rest }}^{a} \\
\mu \mathrm{m}\end{array}$} & \multirow{2}{*}{$\begin{array}{l}\text { Exp. time } \\
{[\mathrm{s}]}\end{array}$} & \multicolumn{2}{|c|}{$F W H M\left[{ }^{\prime \prime}\right]$} & \multirow{2}{*}{$\begin{array}{l}\text { Flux } \\
{[\mathrm{mJy}]}\end{array}$} \\
\hline & & & & & $\operatorname{STD}^{c}$ & Obj. & \\
\hline IC 1459 & $2009-06-05$ & $\begin{array}{c}\text { PAH2 } \\
\text { NeIIref1 }\end{array}$ & $\begin{array}{l}11.18 \pm 0.59 \\
12.20 \pm 0.18\end{array}$ & $\begin{array}{c}362 \\
1443\end{array}$ & $\begin{array}{l}0.29 \\
0.32\end{array}$ & $\begin{array}{l}\ldots \\
\ldots\end{array}$ & $\begin{array}{l}<20 \\
<30\end{array}$ \\
\hline NGC 676 & 2010-12-22 & $\begin{array}{c}\text { PAH2 } \\
\text { PAH2ref2 }\end{array}$ & $\begin{array}{l}11.19 \pm 0.59 \\
11.82 \pm 0.37\end{array}$ & $\begin{array}{l}1448 \\
1448\end{array}$ & $\begin{array}{l}0.32 \\
0.33\end{array}$ & $\begin{array}{l}\ldots \\
\ldots\end{array}$ & $\begin{array}{l}<9 \\
<6\end{array}$ \\
\hline NGC 1097 & $\begin{array}{l}2009-07-14 \\
2006-08-07\end{array}$ & $\begin{array}{l}\text { PAH2 } \\
\text { NeIIref1 }\end{array}$ & $\begin{array}{l}11.20 \pm 0.59 \\
12.22 \pm 0.18\end{array}$ & $\begin{array}{c}997 \\
2524\end{array}$ & $\begin{array}{l}0.39 \\
0.32\end{array}$ & $\begin{array}{l}0.49 \\
0.40\end{array}$ & $\begin{array}{c}60 \pm 10 \\
27 \pm 4\end{array}$ \\
\hline NGC 1404 & $\begin{array}{l}2009-07-15 \\
2009-09-06\end{array}$ & $\begin{array}{c}\text { PAH2 } \\
\text { NeIIref1 }\end{array}$ & $\begin{array}{l}11.18 \pm 0.59 \\
12.19 \pm 0.18\end{array}$ & $\begin{array}{c}544 \\
1992\end{array}$ & $\begin{array}{l}0.57 \\
0.38\end{array}$ & $\begin{array}{l}\cdots \\
\cdots\end{array}$ & $\begin{array}{l}<35 \\
<18\end{array}$ \\
\hline NGC 1566 & 2009-09-06 & $\begin{array}{c}\text { PAH2 } \\
\text { NeIIref1 }\end{array}$ & $\begin{array}{l}11.19 \pm 0.59 \\
12.21 \pm 0.18\end{array}$ & $\begin{array}{c}362 \\
1443\end{array}$ & $\begin{array}{l}0.37 \\
0.33\end{array}$ & $\begin{array}{l}0.46 \\
0.42\end{array}$ & $\begin{array}{c}101 \pm 12 \\
95 \pm 13\end{array}$ \\
\hline NGC 1667 & 2009-09-06 & $\begin{array}{c}\text { PAH2 } \\
\text { NeIIref1 }\end{array}$ & $\begin{array}{l}11.08 \pm 0.58 \\
12.09 \pm 0.18\end{array}$ & $\begin{array}{c}362 \\
1803\end{array}$ & $\begin{array}{l}0.37 \\
0.38\end{array}$ & $\begin{array}{l}\ldots \\
\ldots\end{array}$ & $\begin{array}{l}<16 \\
<15\end{array}$ \\
\hline NGC 3125 & $\begin{array}{l}2009-06-17 \\
2009-06-16\end{array}$ & $\begin{array}{c}\text { PAH2 } \\
\text { NeIIref1 }\end{array}$ & $\begin{array}{l}11.21 \pm 0.58 \\
12.22 \pm 0.18\end{array}$ & $\begin{array}{c}902 \\
1992\end{array}$ & $\begin{array}{l}0.30 \\
0.32\end{array}$ & $\begin{array}{l}0.72 \\
0.74\end{array}$ & $\begin{array}{l}45 \pm 15 \\
65 \pm 13\end{array}$ \\
\hline NGC 3312 & $\begin{array}{c}\text { 2009-07-15 } \\
\ldots\end{array}$ & $\begin{array}{c}\text { PAH2 } \\
\text { NeIIref1 }\end{array}$ & $\begin{array}{l}11.14 \pm 0.59 \\
12.15 \pm 0.18\end{array}$ & $\begin{array}{l}725 \\
\ldots\end{array}$ & $\begin{array}{c}0.38 \\
\ldots\end{array}$ & $\begin{array}{l}\ldots \\
\ldots\end{array}$ & $\begin{array}{l}<24 \\
\ldots\end{array}$ \\
\hline NGC 4235 & 2009-06-07 & $\begin{array}{c}\text { PAH2 } \\
\text { NeIIref1 }\end{array}$ & $\begin{array}{l}11.16 \pm 0.59 \\
12.17 \pm 0.18\end{array}$ & $\begin{array}{c}362 \\
1443\end{array}$ & $\begin{array}{l}0.29 \\
0.33\end{array}$ & $\begin{array}{l}0.38 \\
0.37\end{array}$ & $\begin{array}{l}38 \pm 6 \\
33 \pm 4\end{array}$ \\
\hline NGC 4261 & 2009-06-16 & $\begin{array}{c}\text { PAH2 } \\
\text { NeIIref1 }\end{array}$ & $\begin{array}{l}11.17 \pm 0.59 \\
12.18 \pm 0.18\end{array}$ & $\begin{array}{c}544 \\
1992\end{array}$ & $\begin{array}{l}0.30 \\
0.36\end{array}$ & $\begin{array}{l}0.39 \\
0.48\end{array}$ & $\begin{array}{l}12 \pm 2 \\
15 \pm 2\end{array}$ \\
\hline NGC 4594 (M 104) & 2009-06-17 & $\begin{array}{l}\text { PAH2 } \\
\text { NeIIref1 }\end{array}$ & $\begin{array}{l}11.21 \pm 0.59 \\
12.23 \pm 0.18\end{array}$ & $\begin{array}{l}362 \\
902\end{array}$ & $\begin{array}{l}0.30 \\
0.33\end{array}$ & $\begin{array}{l}\cdots \\
\cdots\end{array}$ & $\begin{array}{l}<11 \\
<17\end{array}$ \\
\hline NGC 4941 & $\begin{array}{l}2009-05-11 \\
2006-04-19\end{array}$ & $\begin{array}{c}\text { PAH2 } \\
\text { NeIIref1 }\end{array}$ & $\begin{array}{l}11.21 \pm 0.59 \\
12.22 \pm 0.18\end{array}$ & $\begin{array}{c}725 \\
1620\end{array}$ & $\begin{array}{l}0.36 \\
0.35\end{array}$ & $\begin{array}{l}0.33 \\
0.35\end{array}$ & $\begin{array}{l}68 \pm 5 \\
75 \pm 4\end{array}$ \\
\hline NGC 5363 & 2009-05-10 & $\begin{array}{c}\text { PAH2 } \\
\text { NeIIref1 }\end{array}$ & $\begin{array}{l}11.21 \pm 0.59 \\
12.22 \pm 0.18\end{array}$ & $\begin{array}{c}544 \\
1443\end{array}$ & $\begin{array}{l}0.33 \\
0.34\end{array}$ & $\begin{array}{l}\cdots \\
\cdots\end{array}$ & $\begin{array}{l}<11 \\
<18\end{array}$ \\
\hline NGC 5813 & 2009-05-10 & $\begin{array}{c}\text { PAH2 } \\
\text { NeIIref1 }\end{array}$ & $\begin{array}{l}11.18 \pm 0.59 \\
12.19 \pm 0.18\end{array}$ & $\begin{array}{l}181 \\
181\end{array}$ & $\begin{array}{l}0.33 \\
0.34\end{array}$ & $\begin{array}{l}\ldots \\
\ldots\end{array}$ & $\begin{array}{l}<21 \\
<51\end{array}$ \\
\hline NGC 7213 & 2009-05-17 & $\begin{array}{l}\text { PAH2 } \\
\text { NeIIref1 }\end{array}$ & $\begin{array}{l}11.18 \pm 0.59 \\
12.20 \pm 0.18\end{array}$ & $\begin{array}{l}181 \\
181\end{array}$ & $\begin{array}{l}0.28 \\
0.31\end{array}$ & $\begin{array}{l}0.30 \\
0.32\end{array}$ & $\begin{array}{c}232 \pm 5 \\
228 \pm 15\end{array}$ \\
\hline NGC 7590 & 2009-06-04 & $\begin{array}{c}\text { PAH2 } \\
\text { NeIIref1 }\end{array}$ & $\begin{array}{l}11.19 \pm 0.59 \\
12.21 \pm 0.18\end{array}$ & $\begin{array}{l}362 \\
902\end{array}$ & $\begin{array}{l}0.32 \\
0.34\end{array}$ & $\begin{array}{l}\ldots \\
\ldots\end{array}$ & $\begin{array}{l}<16 \\
<28\end{array}$ \\
\hline NGC 7626 & 2009-06-04 & $\begin{array}{c}\text { PAH2 } \\
\text { NeIIref1 }\end{array}$ & $\begin{array}{l}11.12 \pm 0.58 \\
12.13 \pm 0.18\end{array}$ & $\begin{array}{l}181 \\
721\end{array}$ & $\begin{array}{l}0.32 \\
0.34\end{array}$ & $\begin{array}{l}\ldots \\
\cdots\end{array}$ & $\begin{array}{l}<24 \\
<26\end{array}$ \\
\hline NGC 7743 & 2009-07-05 & $\begin{array}{c}\text { PAH2 } \\
\text { NeIIref1 }\end{array}$ & $\begin{array}{l}11.19 \pm 0.59 \\
12.20 \pm 0.18\end{array}$ & $\begin{array}{c}362 \\
1443\end{array}$ & $\begin{array}{l}0.29 \\
0.32\end{array}$ & $\begin{array}{l}\ldots \\
\ldots\end{array}$ & $\begin{array}{l}<11 \\
<13\end{array}$ \\
\hline
\end{tabular}

Notes. ${ }^{(a)}$ Central filter wavelength in the restframe with half-band width; ${ }^{(b)}$ on-source exposure time excluding any overheads; ${ }^{(c)} \mathrm{FWHM}$ of the photometric standard star.

target a photometric standard star was observed within $2 \mathrm{~h}$ beforehand or afterward. The observational data are summarized in Table 1.

The observations and MIR properties of the archival objects are described in detail in the literature (Reunanen et al. 2010 for NGC 1386 and NGC 4486; G+09 for ESO 005G004; Horst et al. 2008 for NGC 4303, NGC 4472, NGC 4698, and NGC 5128; and Horst et al. 2006 for NGC 4579), except NGC 1052 (program ID: 382.A-0604, PI: Treister). For all those objects the available raw data were re-reduced in exactly the same way as described below.

Data reductions were performed using the ESO delivered pipeline. Afterward, 2D Gaussians were fitted to all beams of the science and calibration target under the assumption that all targets appear point-like. This will be justified in Sect. 4.1. By subtracting the constant term from the fit, we were able to extract the unresolved flux from the local background. Consequently, this method is not heavily affected by the background as long as it is locally smooth. Owing to the chosen chop throw, the emission structures extended over more than $4^{\prime \prime}$, e.g., the diffuse emission of the host galaxy or weak extended star-formation regions overlap and are at least partially subtracted. Detecting extended sources with VISIR is particularly difficult for the MIR-faint galaxies targeted here. Additional complications arise because of fast local sky background variations of the atmosphere in the MIR and various instrument artifacts that are not understood (see VISIR manual Sect. 4.9).

The absolute photometric calibration was obtained by observing standard stars from Cohen et al. (1999) and computing conversion factors from the integrated intensity of the beams. Its precision is limited by the uncertainties in the fluxes of the standard stars $(\leq 10 \%)$. In addition, a statistical error can be derived by comparing the Gaussian fits of the single beams for each target, and is given in Table 1. Note that a comparison with the aperture method used in Horst et al. (2008) yields consistent results within the uncertainties. 
For non-detected nuclei we performed the following procedure: A 2D Gaussian with the full-width-half-maximum $(F W H M)$ of the standard star and an amplitude of $2 \sigma_{\mathrm{BG}}$ of the local background $(50 \times 50$ pixel $)$ in the image center was integrated, resulting in the flux upper limit. This method was tested and verified by simulating similar beams on very many artificial and real VISIR images. In all cases the artificial beam was clearly visible and these calculated values therefore represent upper limits with a confidence of $\geq 99 \%$. In addition, they agree with expectations based on the exposure time and the sensitivity measured from the standard star during the observations.

\section{Results}

\subsection{MIR morphology and photometry}

Of the 17 observed LLAGN, 7 were detected in both filters. The other 10 and NGC 1404 were not detected in any filter. Corresponding linearly scaled images in the PAH2 and NeIIref1 filters are presented in Fig. 1. These images are created by coadding sub-images $(50 \times 50$ pixels) of the negative beams (with changed sign) to the subimage of the central double beam. In general, all objects show a point-like central source with a detection significance of $\sim 5 \sigma_{\mathrm{BG}}$ in most cases. Here, $\sigma_{\mathrm{BG}}$ signifies the standard variation of the local background ( $\left.4^{\prime \prime}\right)$. The only exception is NGC 3125, which seems to be extended in NeIIref1 and also in PAH2, but the emission is still acceptably fitted by a Gaussian. On the other hand, the detection is at a low $\sigma$-level $(\sim 3)$, which additionally complicates a reliable measurement of its extension. The corresponding $F W H M$ and fluxes as well as upper limits for the non-detections can be found in Table 1. For NGC 1667 individual beams were detected only at a $2 \sigma_{\mathrm{BG}}$-level, but all three beams are located at the expected positions with respect to the chopping parameters. This raises the detection significance to $3.7 \sigma_{\mathrm{BG}}$ for the smoothed combined image (as displayed in Fig. 1). However, the measured fluxes of NGC 1667 will formally be treated as upper limits throughout this work. NGC 676 was not detected, but a foreground star is superposed $5^{\prime \prime}$ south of the galaxy center. The latter was detected in both filters $\left(5 \sigma_{\mathrm{BG}}, 8 \mathrm{mJy}\right.$ in PAH2ref2) constraining the flux of the AGN to $6 \mathrm{mJy}$ in PAh2ref2 ( $3 \sigma_{\mathrm{BG}}$ upper limit). For NGC 4941 and NGC 7213 the Airy rings are evident in the PAH2 images, indicating that the diffraction limit was reached during the exposure in those cases.

Within the pointing accuracy of VISIR all point-source positions are consistent with the ones derived for the galaxy nuclei based on other observations (e.g., 2MASS). We consequently inferred that the observed point-sources are in fact the MIR imprint of the LLAGN. In addition, no other emission sources of any nature are detected within $8^{\prime \prime}$ around the nucleus. Furthermore, the science targets show a $\sim 20 \%$ higher $F W H M$ compared to the standards. This is related to the much longer exposure times of the science observations, which lead to a beam widening caused by two different processes: MIR seeing variations on minute time-scales, and instabilities in the VISIR PSF caused by instrumental effects, as described in Horst et al. (2009). Both effects can lead to a significant variation of the FWHM during long integration times. In principle, this can be quantified and corrected for by comparing and discarding the worst chopping raw frames. However, this is not possible for the LLAGN because they are too faint to be visible in the individual raw frames. Therefore, comparisons between standard and science observations are of limited use even if both were performed under similar atmospheric conditions.

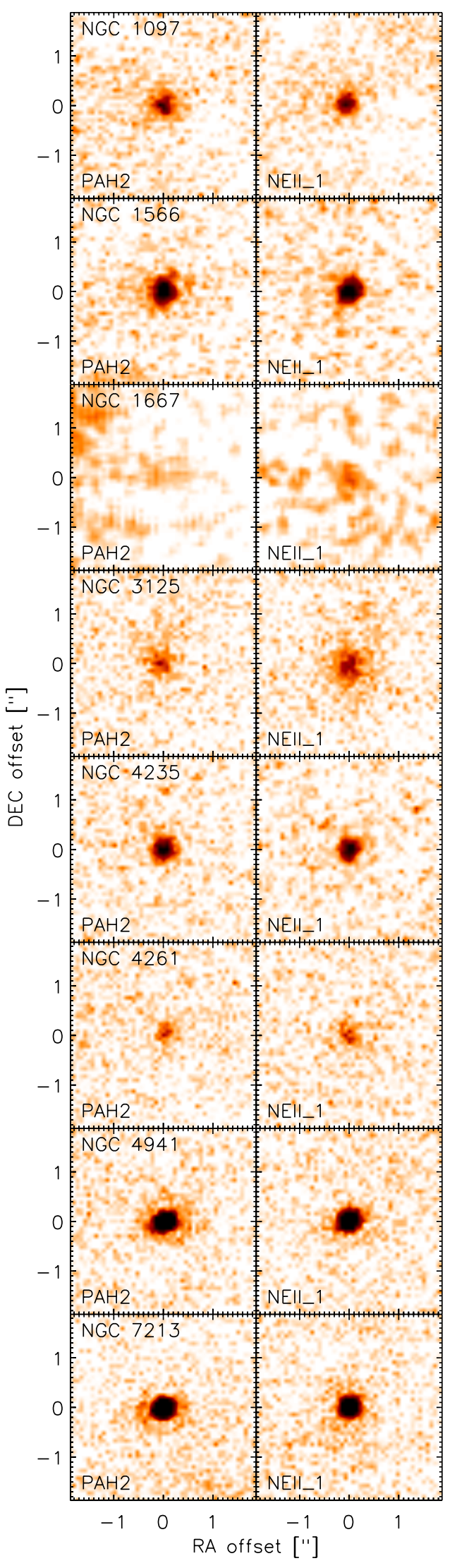

Fig. 1. VISIR images of the P83 LLAGN in the filters PAH2 and NeIIref1. Each row shows one object sorted by right ascension. White corresponds to the mean background value $(\langle\mathrm{BG}\rangle)$ and black color corresponds to $\langle\mathrm{BG}\rangle+10 \sigma_{\mathrm{BG}}$ with a linear scaling. The images of NGC 1667 were smoothed to increase the visibility of this low- $S / N$ detection. 
Table 2. Luminosities and other properties for the whole LLAGN sample.

\begin{tabular}{|c|c|c|c|c|c|c|c|c|c|c|}
\hline Object & AGN type & $\begin{array}{c}D \\
{[\mathrm{Mpc}]}\end{array}$ & Ref. & $\begin{array}{c}r_{0} \\
{[\mathrm{pc}]}\end{array}$ & $\begin{array}{c}\log N_{\mathrm{H}} \\
\mathrm{cm}^{-2}\end{array}$ & $\begin{array}{c}\log L_{2-10 \mathrm{keV}} \\
{[\mathrm{erg} / \mathrm{s}]}\end{array}$ & $\begin{array}{c}\log \lambda L_{12 \mu \mathrm{m}} \\
{[\mathrm{erg} / \mathrm{s}]}\end{array}$ & $\begin{array}{c}\log M_{\mathrm{BH}} \\
{\left[M_{\odot}\right]}\end{array}$ & Ref. & $\log \lambda_{\text {Edd }}$ \\
\hline NGC 676 & Sy 2.0 & $19.5 \pm 8.7$ & 18 & 33 & $>24.3$ & $40.79 \pm 0.60$ & $<40.84$ & 7.56 & 10 & $<-4.0$ \\
\hline NGC 1052 & L & $18.0 \pm 2.4$ & 12 & 31 & 23.1 & $41.18 \pm 0.30$ & $42.13 \pm 0.07$ & 8.19 & 10 & -3.8 \\
\hline NGC 1097 & $\mathrm{~L}$ & $19.1 \pm 4.2$ & 19 & 32 & 20.4 & $40.88 \pm 0.15$ & $41.23 \pm 0.06$ & 8.08 & 13 & -4.2 \\
\hline NGC 1386 & Sy 2.0 & $18.3 \pm 1.4$ & 14 & 31 & $>24.3$ & $42.10 \pm 0.60$ & $42.26 \pm 0.01$ & 7.78 & 11 & -2.9 \\
\hline NGC 1404 & $16 \mathrm{LG}$ & $18.3 \pm 1.4$ & 14 & 31 & $<22.0$ & $39.92 \pm 0.30$ & $<41.25$ & 8.50 & 11 & $<-5.2$ \\
\hline NGC 1566 & Sy 1.5 & $14.3 \pm 5.9$ & 1 & 24 & $<22.0$ & $41.62 \pm 0.30$ & $41.76 \pm 0.06$ & 7.01 & 11 & -2.6 \\
\hline NGC 1667 & Sy 2.0 & $62.6 \pm 4.3$ & 16 & 106 & $>24.3$ & $42.33 \pm 0.60$ & $<42.25$ & 7.81 & 11 & $<-2.8$ \\
\hline ESO 005-G004 & Sy 2.0 & $22.4 \pm 10.0$ & 18 & 38 & 24.0 & $41.82 \pm 0.30$ & $42.00 \pm 0.08$ & 7.89 & 20 & -3.2 \\
\hline NGC 3125 & $\mathrm{~L}$ & $19.8 \pm 1.4$ & 16 & 34 & 21.7 & $40.14 \pm 0.30$ & $41.87 \pm 0.08$ & 5.77 & 5 & -2.0 \\
\hline NGC 3312 & $\mathrm{~L}$ & $44.7 \pm 3.1$ & 16 & 76 & $<22.0$ & $41.10 \pm 0.60$ & $<42.15$ & 8.28 & 11 & $<-3.9$ \\
\hline NGC 4235 & Sy 1.2 & $35.2 \pm 15.7$ & 18 & 60 & 21.2 & $41.61 \pm 0.30$ & $42.08 \pm 0.05$ & 7.60 & 10 & -3.0 \\
\hline NGC 4261 & $\mathrm{~L}$ & $29.4 \pm 2.7$ & 12 & 50 & 23.2 & $41.00 \pm 0.30$ & $41.58 \pm 0.05$ & 8.72 & 6 & -4.7 \\
\hline NGC 4303 (M 61) & Sy 2.0 & $15.2 \pm 3.1$ & 18 & 26 & $<22.0$ & $39.04 \pm 0.30$ & $40.72 \pm 0.23$ & 6.52 & 10 & -3.9 \\
\hline NGC 4472 (M 49) & Sy 2.0 & $17.1 \pm 0.6$ & 15 & 29 & $<22.0$ & $39.00 \pm 0.30$ & $<41.05$ & 8.81 & 10 & $<-6.0$ \\
\hline NGC 4486 (M 87) & L & $17.1 \pm 0.6$ & 15 & 29 & 22.6 & $40.73 \pm 0.30$ & $41.26 \pm 0.05$ & 9.82 & 9 & -6.1 \\
\hline NGC 4579 (M 58) & $\mathrm{L}$ & $16.8 \pm 3.4$ & 18 & 29 & 21.7 & $41.18 \pm 0.15$ & $41.70 \pm 0.06$ & 7.77 & 10 & -3.6 \\
\hline NGC 4594 (M 104) & Sy 1.9 & $9.1 \pm 0.8$ & 12 & 15 & 21.3 & $39.99 \pm 0.30$ & $<40.61$ & 8.46 & 10 & $<-5.4$ \\
\hline NGC 4698 & Sy 2.0 & $23.8 \pm 6.4$ & 17 & 40 & $>24.3$ & $40.82 \pm 0.60$ & $<41.30$ & 7.57 & 10 & $<-3.8$ \\
\hline NGC 4941 & Sy 2.0 & $19.8 \pm 1.4$ & 16 & 34 & 23.7 & $41.43 \pm 0.30$ & $41.94 \pm 0.02$ & 6.91 & 11 & -2.5 \\
\hline NGC 5128 (Cen A) & Sy 2.0 & $3.4 \pm 0.2$ & 7 & 6 & 23.0 & $41.69 \pm 0.15$ & $41.71 \pm 0.01$ & 7.74 & 4 & -3.3 \\
\hline NGC 5363 & $\mathrm{~L}$ & $19.4 \pm 1.4$ & 16 & 33 & $>24.3$ & $41.44 \pm 0.60$ & $<41.30$ & 8.36 & 10 & $<-4.2$ \\
\hline NGC 5813 & $\mathrm{~L}$ & $32.1 \pm 2.8$ & 2 & 54 & 21.1 & $38.77 \pm 0.30$ & $<42.19$ & 8.53 & 10 & $<-5.3$ \\
\hline NGC 7213 & $\mathrm{~L}$ & $21.2 \pm 1.5$ & 16 & 36 & 20.3 & $42.09 \pm 0.15$ & $42.48 \pm 0.03$ & 7.74 & 11 & -2.7 \\
\hline IC 1459 & Sy 2.0 & $30.3 \pm 4.2$ & 2 & 51 & 22.1 & $40.61 \pm 0.30$ & $<41.91$ & 9.41 & 3 & $<-5.4$ \\
\hline NGC 7590 & Sy 2.0 & $27.4 \pm 6.0$ & 19 & 47 & $<22.0$ & $39.77 \pm 0.60$ & $<41.79$ & 6.71 & 8 & $<-3.2$ \\
\hline NGC 7626 & L & $41.9 \pm 2.9$ & 16 & 71 & $<22.0$ & $39.56 \pm 0.30$ & $<42.13$ & 8.78 & 10 & $<-5.2$ \\
\hline NGC 7743 & Sy 2.0 & $19.2 \pm 1.6$ & 12 & 33 & $>24.3$ & $41.27 \pm 0.60$ & $<41.15$ & 6.64 & 10 & $<-2.7$ \\
\hline
\end{tabular}

Notes. References on distances and black hole masses. (1) average of Tully (1988), Willick et al. (1997) and $D_{\mathrm{L}}$ from NED; (2) Blakeslee et al. (2001); (3) Cappellari et al. (2002); (4) Cappellari et al. (2009); (5) Dudik et al. (2005); (6) Ferrarese et al. (1996); (7) Ferrarese et al. (2007); (8) Garcia-Rissmann et al. (2005); (9) Gebhardt et al. (2011); (10) Ho et al. (2009); (11) Hyperleda database (Paturel et al. 2003); (12) Jensen et al. (2003); (13) Lewis \& Eracleous (2006); (14) Madore et al. (1999); (15) Mei et al. (2007); (16) $D_{\mathrm{L}}$ from NED; (17) Springob et al. (2007); (18) Tully (1988); (19) Willick et al. (1997); (29) Winter et al. (2010). $r_{0}$ : spatial scale corresponding to the theoretical diffraction limit for a $8.2 \mathrm{~m}$ VLT telescope at $12 \mu \mathrm{m} . \log \lambda=\log \left(L_{\mathrm{Bol}} / L_{\mathrm{Edd}}\right)$ is the Eddington ratio.

In summary, there is no obvious evidence for extended emission (except NGC 3125), non-nuclear discrete sources or elongation in any LLAGN, but all the observed MIR emission likely originates in a region unresolved by VLT/VISIR.

\subsection{MIR-X-ray relation}

In the following, the P83 data are combined with the archival data described above (Sect. 3) to include all LLAGN detected by VLT/VISIR so far. To compare the positions of the LLAGN in the luminosity plane with respect to the MIR-X-ray correlation, the monochromatic $12 \mu \mathrm{m}$ continuum luminosities $\lambda L_{\lambda}(12 \mu \mathrm{m})$ (hereafter $L_{\mathrm{MIR}}$ ) were computed by using the NeIIref1 fluxes. For this, a flat MIR spectral energy distribution (SED) around $12 \mu \mathrm{m}$ (in $\lambda L_{\lambda}$ ) was assumed and no $K$-correction was applied. Owing to the low redshifts of the sample, the latter effect is negligible. As already described in Sect. 2.2, the intrinsic hard X-ray luminosities $L_{2-10 \mathrm{keV}}$ (hereafter $L_{\mathrm{X}}$ ) are inferred from the literature. The corresponding luminosity values can be found in Table 2 for the distance set c).

The MIR-X-ray correlation strength was measured by using the Spearman rank correlation coefficient, $\rho$, and the corresponding null-hypothesis probability, $p$ ( $r_{-}$correlate in IDL). To quantify the correlation, linear regression in logarithmic space was performed. Because of a variety of systematic uncertainties (see discussion in $\mathrm{G}+09$ ) and a significant number of upper limits, it is useful to compare different fitting methods. Here, the Bayesian based linmix_err (Kelly 2007) and the canonical fi texy (Press et al. 1992), will be used. Both algorithms include treatment of errors on both axes. In addition, linmix_err can handle upper limits and intrinsic scatter as well, which makes it the superior algorithm for this investigation. It uses a Markovchain Monte-Carlo method to draw random parameter sets from the probability distributions given by the measured data. The maxima of the resulting distributions of parameter draws represent the best-fit values (details and comparison tests can be found in Kelly 2007). Here, typically $10^{5}$ of these random draws will be used. Apart from an estimate of the intrinsic scatter $\sigma_{\text {int }}$, linmix_err also returns a linear correlation strength, comparable to the Spearman rank. On the other hand, the Spearman rank and the fitexy will also be stated to facilitate a comparison with previous work. Note that the X-ray luminosity will always be treated as the independent variable. This is physically motivated, because it originates in the innermost region around the black hole directly and traces the accretion, while the MIR emission is likely originated in UV heated dust farther out.

\subsubsection{MIR-X-ray relation for LLAGN}

The absorption-corrected X-ray versus MIR luminosities for all LLAGN are displayed with filled symbols (or arrows in case of upper limits) in Fig. 2 together with the AGN from G+09. To indicate the sensitivity limits caused by the observational layout (see Sect. 2), hatched areas are displayed in the plot as well. 
We were unable to detect objects in those areas at a significant level in the P83 VISIR program. Their borders are determined for each X-ray luminosity by simulating the choice of an exposure time for a hypothetical point-source at a representative distance. This in turn results in a MIR luminosity for which the detection significance would drop below $3 \sigma_{\mathrm{BG}}$. These areas represent only a rough guideline and are not necessarily valid for every individual object, especially in those cases where the exposure times were too short owing to outdated X-ray luminosities (see Sect. 2.2). The latter explains why some upper limits (e.g., NGC 5813 and NGC 7626) are so far above the sensitivity limits.

Although the LLAGN sample does not extend over a wide luminosity range, the MIR and intrinsic X-ray luminosities are highly correlated. However, two outliers become evident by exhibiting offsets $>1$ dex toward high MIR (or low X-ray) luminosities: NGC 3125 and NGC 4303 (solid gray symbols in Fig. 2). Both will be excluded form the correlation and regression analysis, which will be justified in Sect. 5.5. The Spearman rank coefficient varies between 0.78 and 0.88 for the luminosities of the other LLAGN (corresponding to a null-hypothesis probability $-2.54 \geq \log p \geq-3.78$ ), depending on the distance set used. The LLAGN luminosities were fitted with the two algorithms described above, and the fitexy fit to all detected LLAGN is displayed as dashed line in Fig. 2. The results of both fitting algorithms generally agree (compare Table 3), while linmix_err gives systematically flatter slopes and larger uncertainties for the parameters for these samples. Note that the fits including upper limits are likely biased because of the outdated X-ray luminosities which leads to many very high upper limits. Furthermore, the fitexy fit parameters for the different distance sets do not all agree within the calculated uncertainties. Therefore the slope of the MIR-X-ray correlation for LLAGN is generally only constrained to be between $\sim 0.9$ and 1.1. Note that although the Compton-thickness of some objects (e.g., NGC 7590 and NGC 7743) remains uncertain, they do not significantly affect the results. This is because they are not detected with VISIR and their upper limits do not constrain the results with either high (absorbed) or low (unabsorbed) intrinsic $\mathrm{X}$-ray luminosities.

\subsubsection{Comparison to brighter AGN}

The main goal of this investigation is to determine whether LLAGN deviate from the MIR-X-ray correlation found for brighter local AGN (G+09). The latter are plotted in Fig. 2 with empty symbols, while "well-resolved" AGN are additionally marked with a central black-filled circle (see G+09 for details). These "well-resolved" local Seyferts are probably the least contaminated by non-AGN emission, and will be used for comparison with the LLAGN. Their sample properties are repeated in Table 3 and the corresponding power-law fit is also displayed in Fig. 2 as a dotted line. In general, the LLAGN are offset by $\sim 0.3$ dex from the best fit of the brighter AGN toward higher MIR (or lower X-ray) luminosities. In addition, the slopes of the LLAGN fits are all flatter (in the X-ray vs. MIR plane), in particular for the $D_{\mathrm{L}}$ and TF distance sets. However, the uncertainties of the LLAGN fit parameters are so large in all other cases that the 1- $\sigma$ confidence intervals overlap with the brighter AGN fits. Thus, there is no large systematic offset between the LLAGN and AGN. Furthermore, the six detected LINERs constrain any possible difference of this class to the Seyfert class to be $<1$ dex in the MIR-X-ray luminosity plane.

\subsubsection{MIR-X-ray correlation for all AGN}

The previous section showed that the LLAGN do not differ significantly from brighter AGN. Therefore, it makes sense to investigate the properties of the combined AGN sample (LLAGN + all AGN from G+09). The corresponding correlation and regression properties are displayed in the bottom part of Table 3 . The correlation strength measured by the Spearman rank coefficient significantly increases from $\rho=0.88$ to 0.92 and becomes insensitive to the choice of the distance set or even inclusion of the outliers. The same is true also for the fitexy parameters, which are consistent within the uncertainties for all distance sets and sample combinations. In particular, the fit with distance set c) is indistinguishable from the one for all AGN in G+09 (displayed as solid line in Fig. 2). Comparing the fitting algorithms fitexy and linmix_err, the later again gives systematically lower values for the fitted parameters, but consistent with the former.

The MIR-X-ray correlation is also present in the flux-flux space (see Fig. 3). The intrinsic $2-10 \mathrm{keV}$ flux is calculated with the luminosities and distances given in Table 2. Here, the formal correlation strength is weaker $(\rho=0.72 ; \log p=-8.0)$, because of the decreased range in orders of magnitude compared to the luminosity space. The slope flux correlation is very similar to that of the luminosity correlation for the combined sample (using fitexy):

$$
\log \left(\frac{F_{\mathrm{MIR}}}{\mathrm{mJy}}\right)=(14.35 \pm 0.82)+(1.12 \pm 0.08) \log \left(\frac{F_{\mathrm{X}}}{\mathrm{erg} \mathrm{s}^{-1} \mathrm{~cm}^{-2}}\right)
$$

whereas again no $K$-correction is applied. The strong flux correlation and its similar slope prove that the luminosity correlation is not a consequence of a selection bias related to distance.

Summarizing, there is no significant evidence for a deviation of the LLAGN as a group. Instead, they are consistent with the MIR-X-ray luminosity correlation found in previous work for brighter AGN (e.g., G+09). Therefore the main result of this section is that the MIR-X-ray correlation extends unchanged down to luminosities of $\lesssim 10^{41} \mathrm{erg} / \mathrm{s}$ with a small scatter.

\subsubsection{Comparison to starburst galaxies}

It is interesting to compare the MIR-X-ray properties of the AGN to those of typical starburst galaxies (SB) in which the MIR emission is dominated by star-formation. Here, the galaxy sample of Ranalli et al. (2003) is used, consisting of 22 typical nearby starburst galaxies, all possessing measured $2-10 \mathrm{keV}$ $\mathrm{X}$-ray luminosities presented in the same work. The hard X-ray emission in these objects originates mainly in X-ray binaries and shock-heated diffuse gas. Because the star-formation in these galaxies is occurring on global and not (only) nuclear scales, large-aperture photometry is used for the comparison (IRAS fluxes at $12 \mu \mathrm{m}$ ). The SBs are displayed as orange stars in Fig. 2 while the corresponding power-law fit is denoted as the dot-dashed line with parameters listed in Table 3. No luminosity uncertainties are given in Ranalli et al. (2003), therefore we used the bisector algorithm. The SB form a strong correlation $(\rho=0.92 ; \log p=-9.0)$ with a high luminosity ratio $(\bar{r}=3.1)$ and a small scatter $\left(\sigma_{r}=0.25\right)$ in agreement with Krabbe et al. (2001). They form a population clearly distinct from the AGN. Interestingly, the slope $(0.97 \pm 0.07)$ of the power-law fit is comparable to that of the AGN, although the X-ray emission originates from completely different mechanisms. On the other hand, 


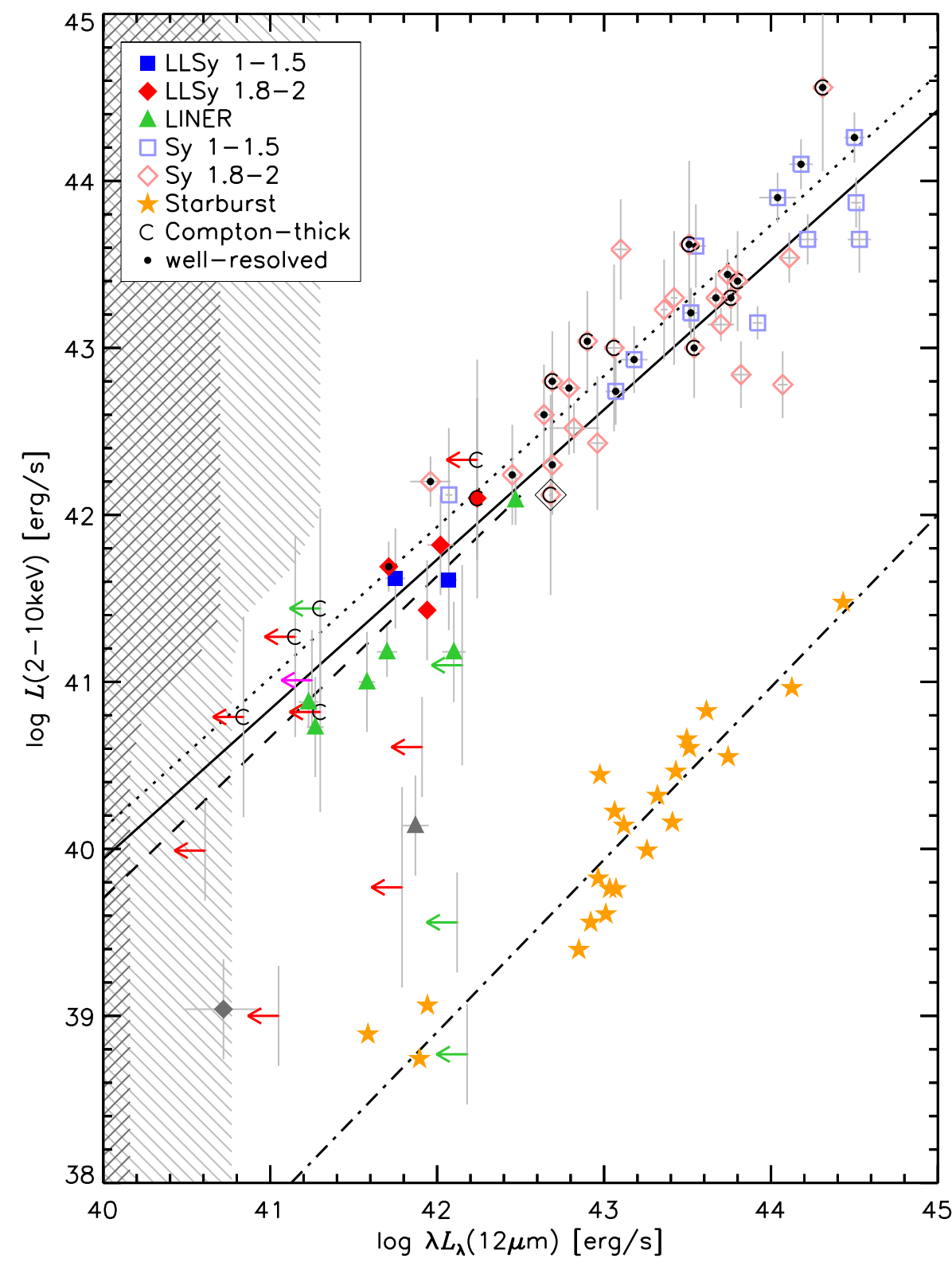

Fig. 2. Absorption-corrected hard X-ray luminosities vs. the nuclear MIR luminosities for all LLAGN (filled symbols) and the AGN from G+09 (empty symbols); blue squares: type 1 Seyferts (type 1.5 or lower), red diamonds: type 2 Seyferts; green triangles: LINERs; magenta arrow: NGC 1404 (NELG); Circinus is plotted for comparison only (framed in black); objects marked with a C: Compton thick AGNs (X-ray $N_{\mathrm{H}}>1.5 \times 10^{24} \mathrm{~cm}^{-2}$ ); objects highlighted with central black-filled circles: "wellresolved" AGN from G+09; NGC 3125 and NGC 4303 are displayed in gray; filled orange stars: starburst galaxies (total luminosities) from Ranalli et al. (2003); solid line: power-law fit to all LLAGN and AGN displayed in color dashed line: power-law fit to all LLAGN (except NGC 3125 and NGC 4303); dotted line: power-law fit to "well-resolved" AGN from $\mathrm{G}+09$; dot-dashed line: power-law fit to all SB. The hatched areas indicate regions below the detection limit of $S / N=3$ for the performed VISIR NeIIref1 imaging: light gray: objects at $20 \mathrm{Mpc}$; dark gray objects at $10 \mathrm{Mpc}$; see text for details. the normalization of the MIR-X-ray correlation for the SB galaxies differs by $\sim 2.6$ orders of magnitude toward higher MIR (or lower X-ray) emission.

\section{Discussion}

\subsection{Comparison with IRAS}

The obtained high spatial resolution data give the opportunity to study the AGN contribution to the total $12 \mu \mathrm{m}$ emission of nearby galaxies. The latter can be measured by using the largeaperture IRAS photometry $\left(F W H M \sim 30^{\prime \prime}\right)$, taken either from Sanders et al. (2003) or the NED database. Figure 4 shows the ratio of the $12 \mu \mathrm{m}$ luminosities measured on a nuclear scale with VISIR and global scale with IRAS over the absorption-corrected X-ray luminosities for all LLAGN and the AGN from G+09. In general, the nuclear fraction of the total MIR luminosity is increasing with increasing X-ray luminosity but with a large scatter of a factor $\sim 2.5$. The two objects with the lowest ratio, NGC 1097 and NGC 4303, are both dusty spiral galaxies, the former dominated by its well-known circum-nuclear starburst ring. Consequently, the total MIR emission of the galaxies is dominated by non-nuclear emission as expected and the AGN contribution dominates only at X-ray luminosities $\gtrsim 10^{43.6} \mathrm{erg} / \mathrm{s}$, while at $L_{X} \sim 10^{42} \mathrm{erg} / \mathrm{s}$ the host contribution to the MIR is already 3 to 10 times higher than that from the nucleus. This was already found by Vasudevan et al. (2010) in the logarithmic space, where a strong correlation $(\rho=0.70, \log p=-6.4)$ is present, and emphasizes once more the need for high spatial resolution to isolate the AGN from surrounding processes.

\subsection{Comparison to Spitzer/IRS}

Most of the LLAGN have also been observed with Spitzer/IRS, which enables a more detailed comparison of the innermost 4 ". In particular, low spectral resolution $N$-band spectra will be used here. Post-BCD spectra are available in the archive, created by a point-source profile extraction. They are well-calibrated (uncertainty $\lesssim 10 \%$ ) and are sufficient for a rough comparison with the nuclear properties, which are the focus of this paper. It is important to note, however, that these IRS spectra can significantly deviate from other studies, because of two possible 
D. Asmus et al.: Mid-infrared properties of nearby low-luminosity AGN at high angular resolution

Table 3. Correlation properties between $\log L_{\mathrm{MIR}}$ and $\log L_{\mathrm{X}}$ for various sample populations.

\begin{tabular}{|c|c|c|c|c|c|c|c|c|c|}
\hline Sample & Method & $N$ & $\rho$ & $\log p$ & $\sigma_{\text {int }}$ & $a$ & $b$ & $\bar{r}$ & $\sigma_{r}$ \\
\hline G+09(well-resolved) & fitexy & 22 & 0.93 & -9.52 & & $0.19 \pm 0.05$ & $1.11 \pm 0.07$ & 0.15 & 0.23 \\
\hline G+09(well-resolved) & linmix_err & 22 & $0.99 \pm 0.02$ & $\ldots$ & $0.12 \pm 0.15$ & $0.18 \pm 0.06$ & $1.08 \pm 0.09$ & 0.15 & 0.23 \\
\hline LLAGN(detected) & fitexy & 12 & 0.78 & -2.54 & & $0.42 \pm 0.27$ & $1.04 \pm 0.17$ & 0.40 & 0.25 \\
\hline LLAGN(detected) & linmix_err & 12 & $0.92 \pm 0.16$ & $\ldots$ & $0.20 \pm 0.25$ & $0.16 \pm 0.44$ & $0.85 \pm 0.28$ & 0.40 & 0.25 \\
\hline LLAGN(all) & linmix_err & 25 & $0.98 \pm 0.05$ & & $0.25 \pm 0.36$ & $0.44 \pm 0.46$ & $1.09 \pm 0.27$ & 0.40 & 0.25 \\
\hline LLAGN(detected; $D_{\mathrm{L}}$ ) & fitexy & 12 & 0.88 & -3.78 & $\ldots$ & $0.06 \pm 0.12$ & $0.74 \pm 0.08$ & 0.42 & 0.25 \\
\hline LLAGN(detected; TF) & fitexy & 12 & 0.84 & -3.14 & $\ldots$ & $0.15 \pm 0.17$ & $0.84 \pm 0.10$ & 0.42 & 0.25 \\
\hline LINER(detected) & fitexy & 6 & 0.93 & -2.12 & & $0.49 \pm 0.32$ & $1.01 \pm 0.17$ & 0.55 & 0.20 \\
\hline LINER(detected) & linmix_err & 6 & $0.89 \pm 0.34$ & $\ldots$ & $0.34 \pm 2.76$ & $0.39 \pm 1.49$ & $0.94 \pm 0.81$ & 0.55 & 0.20 \\
\hline LINER(all) & linmix_err & 10 & $0.94 \pm 0.24$ & $\ldots$ & $0.38 \pm 5.39$ & $0.39 \pm 2.00$ & $0.96 \pm 1.10$ & 0.55 & 0.20 \\
\hline $\mathrm{G}+09$ (all) & fitexy & 42 & 0.88 & -14.01 & & $0.41 \pm 0.03$ & $1.12 \pm 0.04$ & 0.30 & 0.36 \\
\hline $\mathrm{G}+09$ (all) & linmix_err & 42 & $0.95 \pm 0.03$ & $\ldots$ & $0.29 \pm 0.19$ & $0.35 \pm 0.06$ & $1.00 \pm 0.08$ & 0.30 & 0.36 \\
\hline LLAGN(detected) \& G+09(all) & fitexy & 48 & 0.92 & -19.02 & & $0.41 \pm 0.03$ & $1.12 \pm 0.04$ & 0.31 & 0.35 \\
\hline LLAGN(detected) \& G+09(all) & linmix_err & 48 & $0.96 \pm 0.02$ & $\ldots$ & $0.26 \pm 0.17$ & $0.35 \pm 0.06$ & $1.02 \pm 0.06$ & 0.31 & 0.35 \\
\hline LLAGN(all) \& G+09(all) & linmix_err & 61 & $0.98 \pm 0.01$ & $\ldots$ & $0.27 \pm 0.17$ & $0.34 \pm 0.06$ & $1.06 \pm 0.06$ & 0.31 & 0.35 \\
\hline $\mathrm{SB}$ & bisector & 22 & 0.92 & -8.97 & $\ldots$ & $2.96 \pm 0.20$ & $0.97 \pm 0.07$ & 3.06 & 0.25 \\
\hline
\end{tabular}

Notes. Methods: see text for a description; for "linmix_err" the given values are medians of the parameters; all uncertainties are given as standard deviations; $N$ : number of objects used for the analysis; $\rho$ : linear correlation coefficient (for "fitexy": Spearman Rank); $p$ : null-hypothesis probability; $\sigma_{\text {int }}$ : intrinsic scatter (estimated by "linmix_err"); $a, b$ : fitting parameters of $\log L_{\mathrm{MIR}}-43=a+b\left(\log L_{\mathrm{X}}-43\right) ; \bar{r}$ : average of the luminosity ratio $r=\log \left(L_{\mathrm{MIR}} / L_{\mathrm{X}}\right)$ with $\sigma_{r}$ its standard deviation.

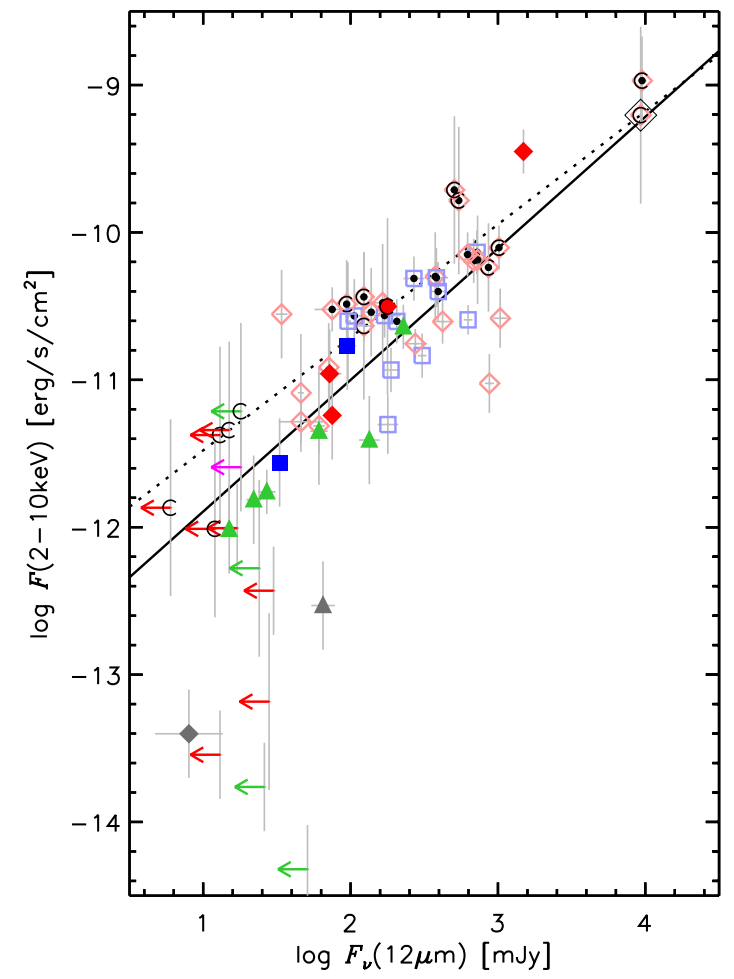

Fig. 3. Correlation of MIR and absorption-corrected hard X-ray fluxes for the extended LLAGN sample and AGN from G+09. Description is similar as in Fig. 2.

reasons: for extended objects the extraction profile underweight the extended emission, or the flux might be significantly lower because of bigger extraction areas (e.g., 20" in Gallimore et al. 2010). Nevertheless, for the objects in common, NGC 1097 and NGC 1566, the post-BCD spectra generally agree with those published by Diamond-Stanic \& Rieke (2010), where very small extraction areas have been used.

The data are displayed in Fig. 5 without the flux uncertainties of the IRS spectra $(\lesssim 10 \%)$ for clarity. Common MIR emission

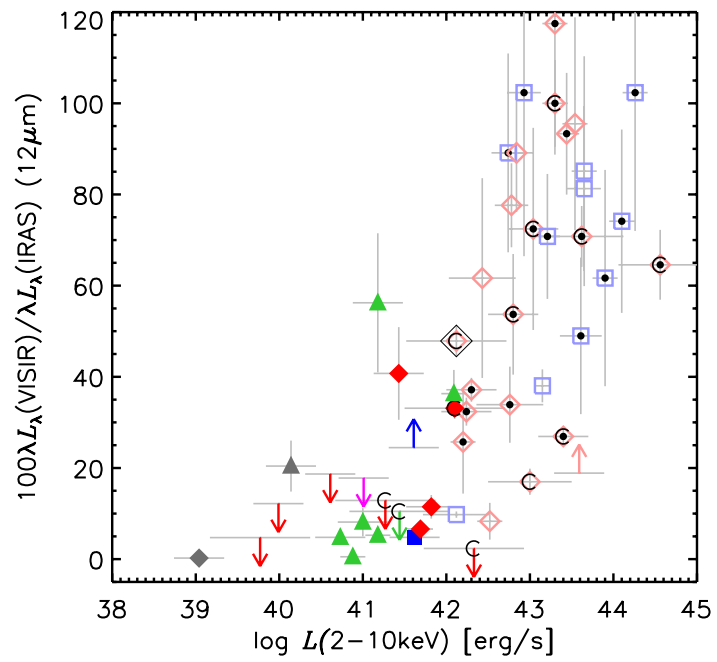

Fig. 4. Fraction of total $12 \mu \mathrm{m}$ luminosity that is emitted by the nucleus. It is measured by the ratio of VISIR to IRAS luminosities versus the absorption-corrected hard X-ray luminosity for the extended LLAGN sample and AGN from G+09. Description is similar as in Fig. 2, except for the dashed line, which is a power-law fit (linmix_err) to the combined AGN sample.

lines are indicated by vertical dashed lines, from left to right: [ArIII] $(8.99 \mu \mathrm{m}), \mathrm{H}_{2}(9.67 \mu \mathrm{m})$, [SIV] $(10.5 \mu \mathrm{m}), \mathrm{PAH}$ $(11.3 \mu \mathrm{m})$ and [NeII] $(12.81 \mu \mathrm{m})$. For NGC 676, NGC 3312, and NGC 5363 no IRS spectra are available in the archive. Furthermore, for NGC 1667, NGC 4594, NGC 7590 and NGC 7626, the IRS spectra are in high-resolution mode with a low $S / N$, although in some cases a strong PAH feature $(11.3 \mu \mathrm{m})$ is still evident. In addition, archival VISIR imaging data along with the photometric measurements of Ramos Almeida et al. (2009) and Mason et al. (2007) are shown for comparison (gray data points). They agree well with the new measurements within the uncertainties. Only NGC 7213 shows different fluxes ( $\gtrsim 10 \%)$ in both NeIIref1 and PAH2 over a time of about three years. The reason for this is unknown but might indicate variability. 

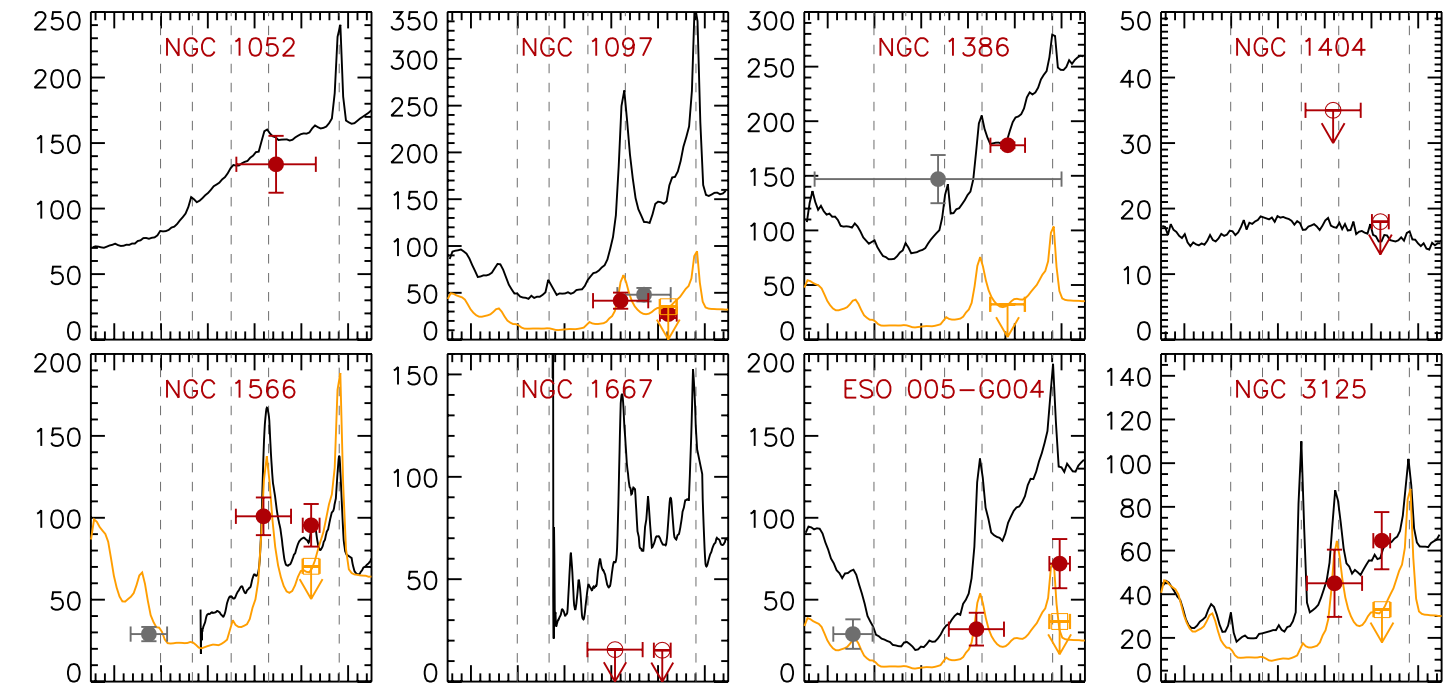

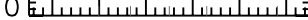

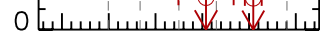
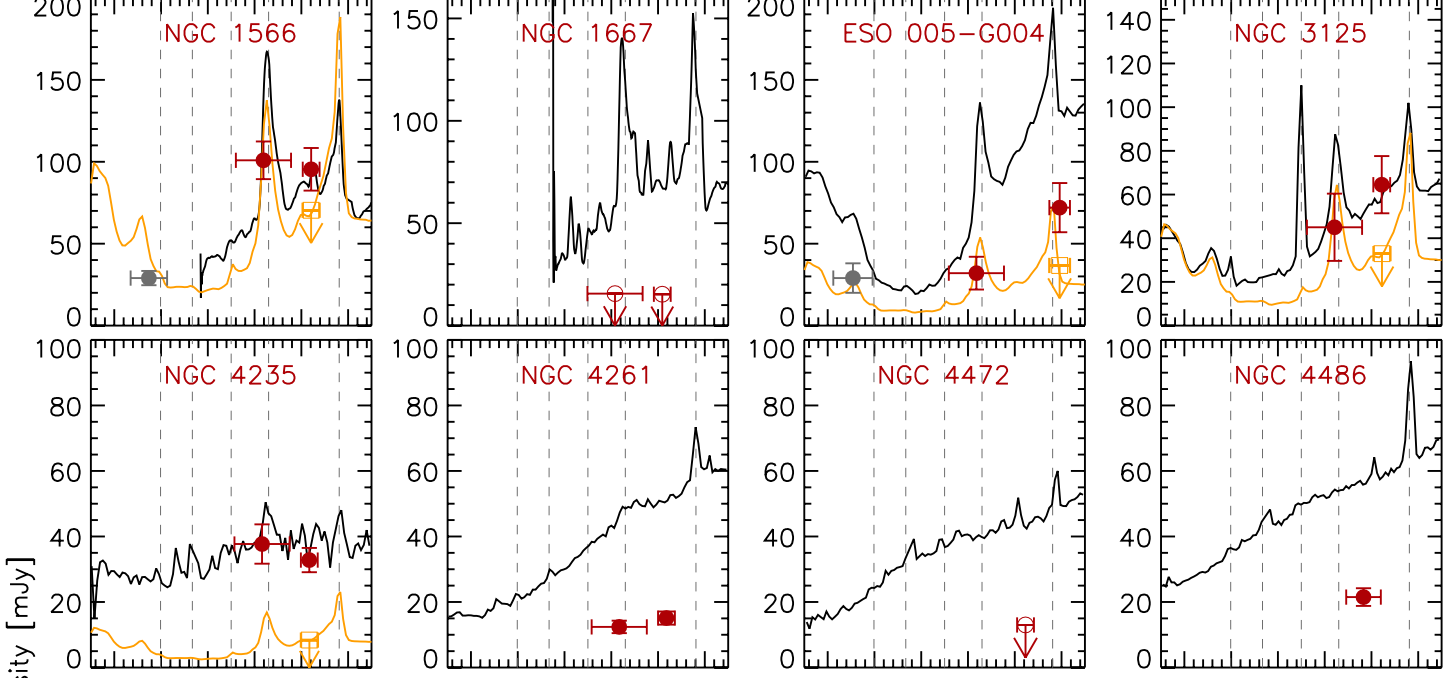

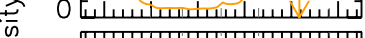
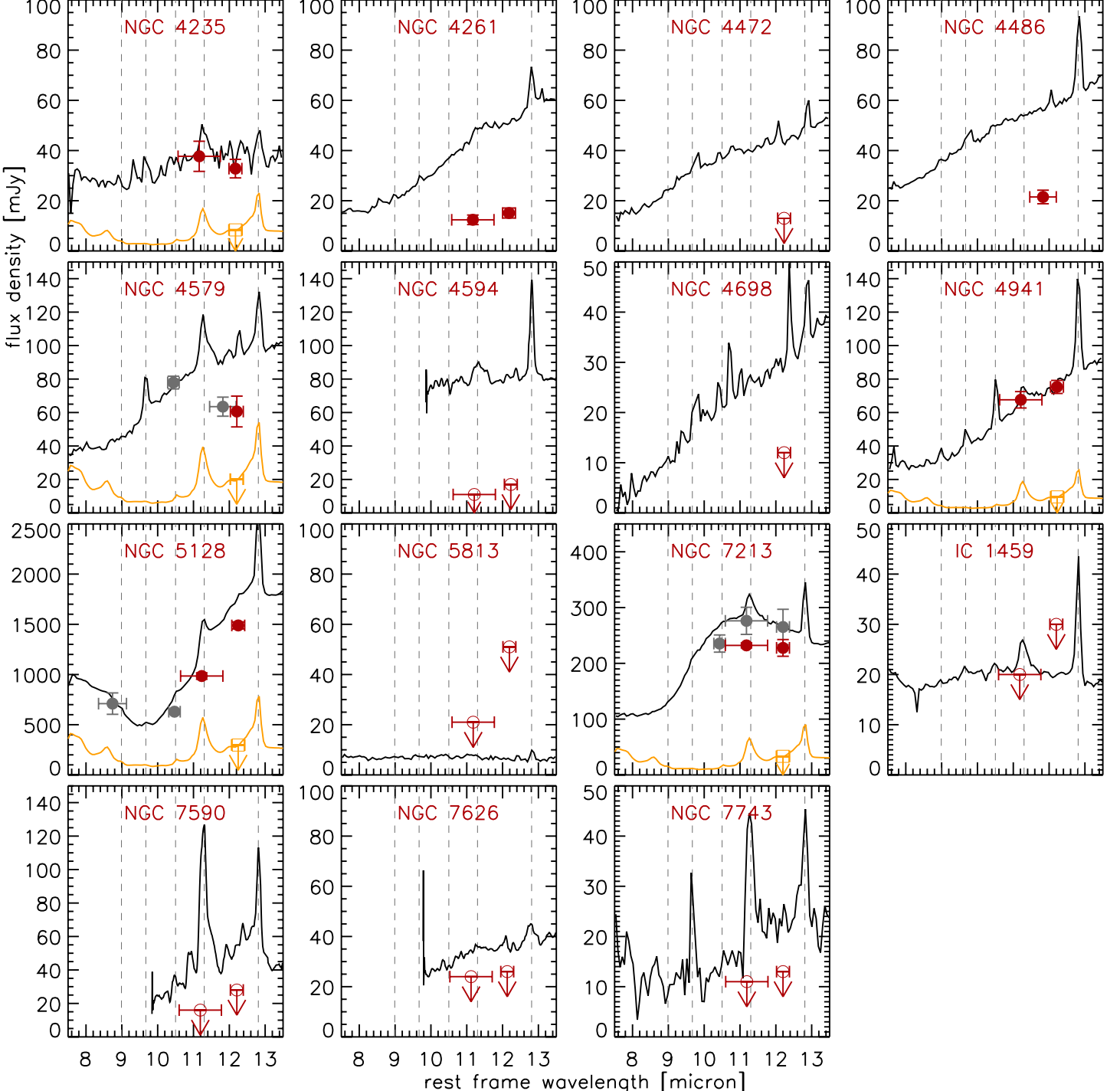

Fig. 5. Comparison of VISIR photometry (red symbols: P83 data, gray symbols: re-reduced data from the archive) and Spitzer IRS spectra (black line). In addition, photometric measurements in the narrow-N filter for NGC 1097 (Mason et al. 2007), and the Si2 filter for NGC 1386 and NGC 5128 and in the N filter for NGC 1386 are also plotted in gray for comparison (Ramos Almeida et al. 2009). Horizontal error bars correspond to the filter pass band. The IRS spectra of NGC 1566, NGC 4594, NGC 1667, NGC 7590, and NGC 7626 are smoothed for better visibility. Common emission lines are indicated by the dotted lines. We used the redshifts from the NED database for the rest frame wavelengths. In addition, the scaled starburst template SED (displayed as orange line) are shown as well as the derived upper limit for the starburst contribution to the continuum filter measurement (orange symbols), see Sect. 5.3 for details. 
Table 4. Comparison between VISIR and Spitzer/IRS flux densities.

\begin{tabular}{lcccccc}
\hline \hline Object & $\begin{array}{c}F_{v}^{\mathrm{VISIR}}(\mathrm{PAH} 2) \\
{[\mathrm{mJy}]}\end{array}$ & $\begin{array}{c}F_{v}^{\mathrm{IRS}}(\mathrm{PAH} 2) \\
{[\mathrm{mJy}]}\end{array}$ & $R$ (PAH2) & $\begin{array}{c}F_{v}^{\mathrm{VISIR}}(\mathrm{NeIIref1}) \\
{[\mathrm{mJy}]}\end{array}$ & $\begin{array}{c}F_{v}^{\mathrm{IRS}}(\mathrm{NeIIref1}) \\
{[\mathrm{mJy}]}\end{array}$ & $R$ (NeIIref1) \\
\hline NGC 1097 & $60.4 \pm 10.2$ & 168.4 & 2.8 & $27.3 \pm 4.2$ & 158.2 & 5.8 \\
NGC 1566 & $100.9 \pm 11.5$ & 103.2 & 1.0 & $95.4 \pm 13$ & 94.0 & 1.0 \\
NGC 3125 & $45 \pm 15.4$ & 60.2 & 1.3 & $64.5 \pm 13.1$ & 59.2 & 0.9 \\
NGC 4235 & $37.7 \pm 6$ & 41.1 & 1.1 & $32.8 \pm 3.7$ & 39.6 & 1.2 \\
NGC 4261 & $11.8 \pm 2$ & 46.0 & 3.9 & $15.1 \pm 2$ & 51.2 & 3.4 \\
NGC 4941 & $67.6 \pm 4.9$ & 68.0 & 1.0 & $75.3 \pm 3.9$ & 76.5 & 1.0 \\
NGC 5128 & $984.8 \pm 32.1$ & 1322.9 & 1.3 & $1488.7 \pm 35.1$ & 1767.7 & 1.2 \\
NGC 7213 & $232.2 \pm 4.5$ & 297.5 & 1.3 & $227.7 \pm 15$ & 263.2 & 1.2 \\
\hline
\end{tabular}

Notes. The given IRS fluxes are extracted from the BCD spectra convolved with the normalized VISIR filter transfer functions of PAH2 and NeIIref1; the PAH2 fluxes contain not only the PAH feature at $11.3 \mu \mathrm{m}$ but also the underlying continuum; $R(\mathrm{PAH} 2)$ and $R(\mathrm{NeIIref1)}$ give the flux ratios $\left(F_{v}^{\mathrm{IRS}} / F_{v}^{\mathrm{VISIR}}\right)$ for both filters.

For comparison with the VISIR photometry $\left(F_{v}^{\mathrm{VISIR}}\right)$, the IRS spectra were convolved with the normalized filter transfer functions of PAH2 and NeIIref1. The resulting fluxes, $F_{v}^{\mathrm{IRS}}$ (PAH2) and $F_{v}^{\mathrm{IRS}}($ NeIIref1), are displayed in Table 4 for all LLAGN detected in both filters. There are many cases where Spitzer displays a much higher $12 \mu \mathrm{m}$ continuum flux than VISIR at a higher spatial resolution, especially for NGC 1097, NGC 4261 (also NGC 4486). This is also true for the upper limits derived for NGC 1667, NGC 4594 and NGC 7590 and implies that large amounts of hot dust are distributed in extended diffuse regions around the nucleus. Owing to their faintness, the latter are probably resolved-out in VISIR and consequently are not visible. Unfortunately, the $S / N$ of the images is not high enough to constrain these emission regions well, e.g., the missing flux of $\sim 35$ mJy of NGC 4261 extended over a region of $0.75^{\prime \prime}$ diameter would become undetectable in VISIR. However, for the other objects (NGC 1566, NGC 3125, NGC 4235 and NGC 4941) the VISIR and IRS continuum fluxes are very similar, indicating that in these cases no additional significant MIR source surrounds the nucleus at arcsec scale.

The imaging in the second filter setting (PAH2) was performed to constrain the strength of the PAH emission feature at $11.3 \mu \mathrm{m}$, which is commonly used as a star-formation indicator (e.g., Diamond-Stanic \& Rieke 2011). The convolved IRS flux $\left(F_{v}^{\mathrm{IRS}}(\mathrm{PAH} 2)=103 \pm 10 \mathrm{mJy}\right)$ for NGC 1566 is particularly similar to the VISIR PAH2 flux $\left(F_{v}^{\mathrm{VISIR}}(\mathrm{PAH} 2)=100.9 \pm 11.5 \mathrm{mJy}\right)$. This indicates the presence of PAH emission inside the inner $0.4^{\prime \prime}$. On the other hand, the weak PAH emission features present in the IRS spectra of NGC 4235 and NGC 4941 cannot be verified in the VISIR data because the measurement uncertainties are too large. For NGC 1097, the VISIR fluxes are both much lower than in the IRS spectrum - the latter showing very strong PAH emission. However, the PAH2 flux in VISIR is much higher than in NeIIref1, implying that the nucleus of NGC 1097 also emits large amounts of PAH emission. Nevertheless the lower continuum fluxes and absence of PAH emission in VISIR indicates in general that this feature, which is typical for starformation, usually does not originate in the very nucleus of LLAGN, but in the circum-nuclear surroundings (at scales larger than $100 \mathrm{pc}$ ). This was already noticed by Hönig et al. (2010) for Seyfert galaxies. These diffuse emission regions are likely very extended, so that they are resolved-out on most VISIR images.

We will examine in the next section the most extreme possible case, i.e., that all PAH emission visible in the IRS spectra originates in the unresolved nucleus, to constrain any starformation contamination of the VISIR data even better.

\subsection{Constraining the nuclear star-formation contamination}

One of our main concerns is whether the measured $12 \mu \mathrm{m}$ continuum is contaminated by non-AGN emission, e.g., circumnuclear star-formation. This contamination would increasingly affect the measurements at lower luminosities as in the case of the LLAGN. It could indeed explain the MIR excess that some LLAGN show. To constrain the individual star-formation contamination better, the LLAGN data can be compared to a typical star-formation SED. This template SED can be created by using, e.g., the IRS spectra of typical nearby starburst galaxies from Brandl et al. (2006). These authors collected a sample of $13 \mathrm{SB}$ spectra with highest $S / N$. They have been made available electronically by the Spitzer/IRS Atlas project (HernánCaballero \& Hatziminaoglou 2011). To create the SB template SED, these $13 \mathrm{SB}$ are normalized by their PAH $11.3 \mu \mathrm{m}$ emission line flux. This approach is valid because the $12 \mu \mathrm{m}$ continuum scales with the PAH $11.3 \mu \mathrm{m}$ flux in starbursts as demonstrated by a strong correlation for the whole sample of Brandl et al. (2006) $(\rho=0.98$ with $\log p=-7.1)$ :

$$
\begin{aligned}
\log \left(\frac{F_{v}(\text { NeIIref } 1)}{\mathrm{Jy}}\right) & =(11.82 \pm 0.91) \\
& +(1.06 \pm 0.08) \log \left(\frac{F(\text { PAH } 11.3 \mu \mathrm{m})}{\mathrm{erg} \mathrm{s}^{-1} \mathrm{~cm}^{-2}}\right)
\end{aligned}
$$

A comparable correlation was recently found by Diamond-Stanic \& Rieke (2011). Note that this normalization results in a scatter of approximately $30 \%$ ( $0.13 \mathrm{dex})$ for the $12 \mu \mathrm{m}$ flux in the individual spectra. The created SB template SED is then scaled for each individual LLAGN by its PAH $11.3 \mu \mathrm{m}$ emission line flux. Next, the corresponding $12 \mu \mathrm{m}$ flux of the scaled SB is determined by convolution with the continuum filter function (NeIIref1 in most cases). By this means it is possible to estimate the maximum contamination by star-formation in the measured continuum of the unresolved LLAGN nuclei in VISIR.

The measured total PAH $11.3 \mu \mathrm{m}$ emission line fluxes $F(\mathrm{PAH} 11.3 \mu \mathrm{m})$ are given in Table 5 for all detected LLAGN with evident PAH emission, while the typical measurement error is $\lesssim 10 \%$. The scaled starburst template spectra for those LLAGN are plotted in orange in Fig. 5. The corresponding calculated $12 \mu \mathrm{m}$ continuum fluxes $F_{v}^{\mathrm{SB}}(12 \mu \mathrm{m})$ are indicated by orange open squares and stated in Table 5 as well. Note that NGC 1052 is not included because it was not observed in a VISIR filter containing only continuum, but instead in the B11.4 filter. Although this filter includes the PAH emission feature in its 
Table 5. Estimate of maximum star formation contribution.

\begin{tabular}{lccc}
\hline \hline Object & $\begin{array}{c}\log F(\mathrm{PAH} 11.3 \mu \mathrm{m}) \\
{\left[\mathrm{erg} / \mathrm{s} / \mathrm{cm}^{2}\right]}\end{array}$ & $\begin{array}{c}F_{v}^{\mathrm{SB}}(12 \mu \mathrm{m}) \\
{[\mathrm{mJy}]}\end{array}$ & $\begin{array}{c}c_{<}^{\mathrm{SB} a} \\
\%\end{array}$ \\
\hline NGC 1097 & -11.95 & $35.2^{b}$ & 100 \\
NGC 1386 & -12.47 & 32.3 & 18 \\
NGC 1566 & -12.21 & 70.4 & 73 \\
ESO 005-G004 & -12.37 & $36.7^{c}$ & 50 \\
NGC 3125 & -12.54 & 32.9 & 51 \\
NGC 4235 & -13.13 & 8.4 & 25 \\
NGC 4579 & -12.76 & 20.2 & 33 \\
NGC 4941 & -13.08 & 9.6 & 12 \\
NGC 5128 & -11.59 & 297.4 & 19 \\
NGC 7213 & -12.53 & 33.3 & 14 \\
\hline
\end{tabular}

Notes. ${ }^{(a)} c_{<}^{\mathrm{SB}}$ signifies the maximum star formation contribution (in percent) to the nuclear $12 \mu \mathrm{m}$ continuum flux (at $0.4^{\prime \prime}$ scale). ${ }^{(b)} F_{v}^{\mathrm{SB}}(12 \mu \mathrm{m})$ of NGC 1097 constrained by the VISIR PAH flux, see text for details. (c) $F_{v}^{\mathrm{SB}}(12 \mu \mathrm{m})$ of ESO 005-G004 constrained by the VISIR PAH2 flux, see text for details.

band-pass, the emission is very weak in the IRS spectrum of NGC 1052. Furthermore, no significant PAH emission could be detected in the IRS spectra for the detected LINERs NGC 4261 and NGC 4486. This makes any significant contribution of starformation to the VISIR data of NGC 1052, NGC 4261 and NGC 4486 very unlikely. On the other hand, the IRS spectra of NGC 1097 and NGC 1566 show strong PAH emission, which means that their estimated maximum star-formation contribution $c_{<}^{\mathrm{SB}}$ is very high (100\% and $73 \%$ respectively). Note, however, that in the case of NGC 1097 and ESO 005-G004, $F_{v}^{\mathrm{SB}}(12 \mu \mathrm{m})$ was actually constrained more by the VISIR PAH2 flux, because this was lower than the scaled flux of the SB template. Nevertheless, in the case of NGC 1097, $F_{v}^{\mathrm{SB}}(12 \mu \mathrm{m})$ still exceeds the measured $12 \mu \mathrm{m}$ continuum flux in VISIR. However, the PAH emission feature is weak in all other cases (NGC 1386, NGC 4235, NGC 4579, NGC 4941, NGC 5128 and NGC 7213), so that the maximum star-formation contribution $c_{<}^{\mathrm{SB}}$ can be constrained often to much less than $30 \%$. In summary, a major contribution by star-formation to the nuclear $12 \mu \mathrm{m}$ continuum (at $0.4^{\prime \prime}$ scale) can be excluded for the majority of LLAGN (9 of 12 cases). Note that exclusion of the other three possibly contaminated LLAGN does not affect the MIR-X-ray correlation.

However, it is very unlikely that the entire PAH emission originates in the unresolved nucleus (as assumed in this section). Consequently, the real star-formation contribution is probably much lower in all cases, as long as the PAH $11.3 \mu \mathrm{m}$ remains a valid tracer of the star-formation. It is not verified that the hard AGN radiation not only destroys the PAH molecules but also quenches star-formation. On the other hand, PAH molecules might survive in the dense clumpy clouds inside the torus itself for significant timescales. In addition, young massive star clusters emit hard radiation fields that could destroy the PAH molecules (see e.g., Snijders et al. 2006). At least, recent findings suggest that the PAH $11.3 \mu \mathrm{m}$ remains on average a good star-formation tracer also in circum-nuclear environments of AGN (e.g., Diamond-Stanic \& Rieke 2010).

\subsection{Accretion rates}

After we showed that most LLAGN are not dominated by nuclear star-formation in the MIR, we now study the relation between the MIR emission and other AGN properties, specifically the accretion rate. Here, the Eddington ratio will be used as a proxy of the accretion rate: $\lambda_{\mathrm{Edd}}=L_{\mathrm{Bol}} / L_{\mathrm{Edd}}$ with $L_{\mathrm{Bol}}$ the bolometric luminosity and $L_{\mathrm{Edd}}=1.26 \times 10^{38}\left(M_{\mathrm{BH}} / M_{\odot}\right) \mathrm{erg} / \mathrm{s}$ being the Eddington luminosity. The X-ray luminosity is often used to estimate $L_{\text {Bol }}$ (e.g., Vasudevan \& Fabian 2007; Vasudevan et al. 2009) where $L_{\mathrm{Bol}}=10 L_{2-10 \mathrm{keV}}$ is a reasonable choice, whereas the exact value of the bolometric correction factor does not affect the main conclusions of this study. The black hole masses used in this analysis were compiled from the literature with preference given to the most recent determinations. We list the masses in Tables 2 and A.1 for the AGN from G+09. In most cases the only available mass estimate comes from using the empirical $M_{\mathrm{BH}}-\sigma_{*}$ relation (Gebhardt et al. 2000; Ferrarese \& Merritt 2000; Tremaine et al. 2002), and in those cases the given reference is for the stellar velocity $\sigma_{*}$. Furthermore, the newest determination of the relation (Gültekin et al. 2009) is used, for which the intrinsic scatter is estimated to be 0.44 dex. Here, this value will be adopted as general uncertainty for $M_{\mathrm{BH}}$.

Figure 6 shows the MIR-X-ray luminosity ratio, $r=$ $\log \left(L_{\mathrm{MIR}} / L_{\mathrm{X}}\right)$, distribution versus the computed accretion rate. In general, the observed LLAGN have low accretion rates $\left(\lambda_{\text {Edd }} \leq-2.5\right)$, whereas the low-luminosity Seyferts overlap with the brighter AGN. On the other hand, the LINERs become clearly separated with lowest accretion rates. These are believed to be a critical parameter determining the AGN structure. As already mentioned, for low accretion rates several changes in the AGN structure are suggested, e.g., for the accretion disk. In addition, Falcke \& Markoff (2000) argued that the jet might dominate the AGN emission at low accretion rates and presented a jet model that explains the observed SED of Sgr A*. A similar model was used by Markoff et al. (2008) to fit the extensive multiwavelength data of M 81 successfully, a well-known LLAGN. These changes in the AGN structure will also be reflected in the mid-infrared. Although neither the accretion disk nor the jet base can be resolved with VLT/VISIR, if any of the two dominates the nuclear MIR emission, evidence should be present in the data. In particular, Yuan \& Cui (2005) predict that the jet (if present) should dominate the X-ray emission at rates below $\lambda_{\mathrm{Edd}}^{\text {crit }} \approx 10^{-5}$. This is for example the case for NGC 4486, NGC 4594 and IC 1459, while NGC 1052, NGC 4261 and NGC 4579 should be ADAF dominated. All these objects are also included in the sample of Yuan et al. (2009), who have successfully tested the above prediction by SED fitting. These authors did not include a torus model and instead the MIR emission is mainly produced by the ADAF. In this case, the MIR-X-ray luminosity ratio is predicted to be roughly one for the ADAF-dominated sources and is significantly higher for the jet-dominated sources NGC 4486 and NGC 4594. But this difference in the ratios is not evident in Fig. 6.

In general, the average luminosity ratio is higher for LLAGN $(\bar{r}=0.40 \pm 0.25)$ compared to the values for the well-resolved $(0.15 \pm 0.23)$ and for all AGN from $\mathrm{G}+09(0.30 \pm 0.36$; see also Table 3$)$. In particular, LINERs have a high luminosity ratio $(\bar{r}=$ $0.55 \pm 0.20)$ compared to type $1(\bar{r}=0.34 \pm 0.29)$ and type 2 Seyferts $(\bar{r}=0.23 \pm 0.38)$. However, because of the large scatter any differences remain statistically insignificant. As expected, NGC 4303 and NGC 3125 are located at peculiar positions in this graph, and especially the latter possesses an atypically high accretion rate for a LINER $\left(\lambda_{\mathrm{Edd}}=10^{-2}\right)$. In summary, despite the different object types the MIR-X-ray luminosity ratio can be described as constant for all AGN with a scatter of $0.35 \mathrm{dex}$ over at least 4 (possibly 6) orders of magnitude in accretion rate $\left(10^{-6} \lesssim \lambda_{\text {Edd }} \lesssim 1\right)$. 


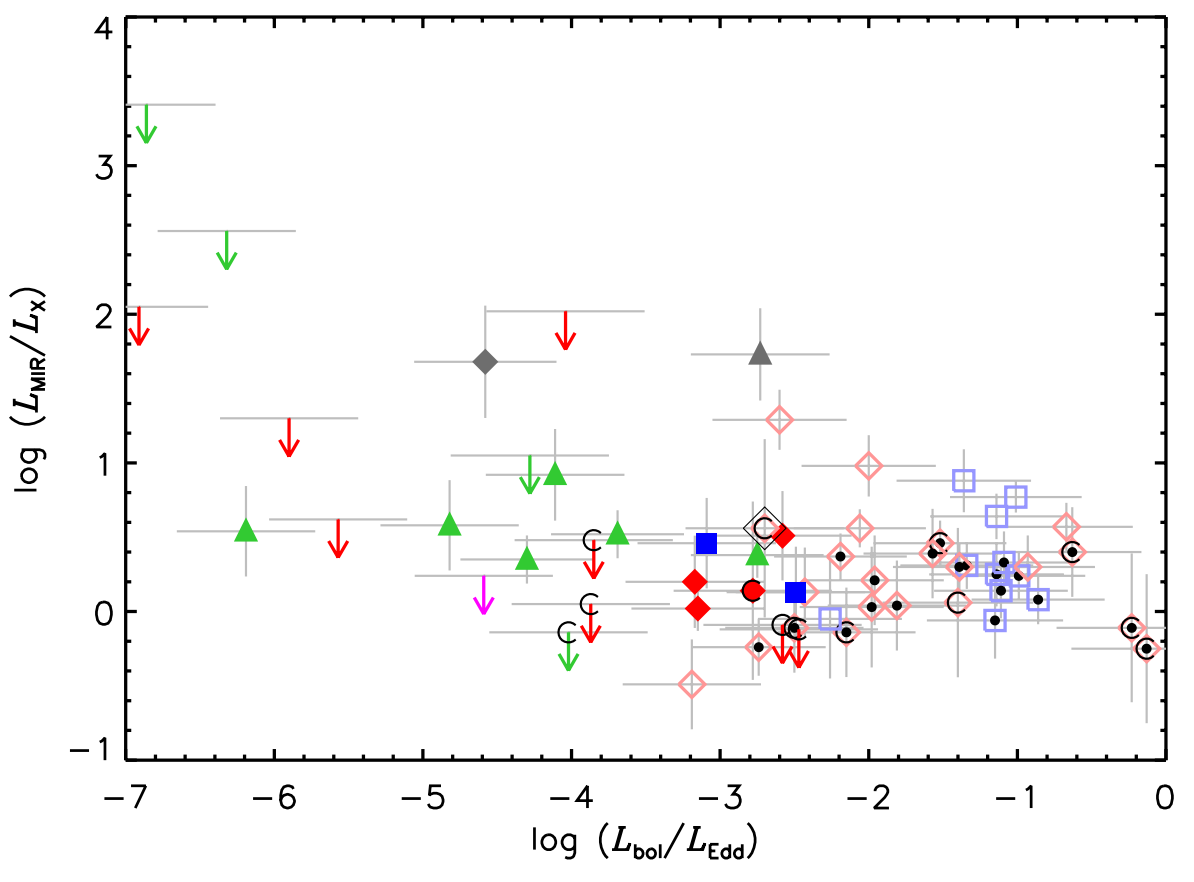

Fig. 6. $12 \mu \mathrm{m}$ to $2-10 \mathrm{keV}$ luminosity ratio versus Eddington ratio $\log \lambda_{\mathrm{Edd}}=\log \left(L_{\mathrm{Bol}} / L_{\mathrm{Edd}}\right)$ for all AGN with available black hole mass estimates. Symbols are as in Fig. 2.

\subsection{The outliers}

Two of the objects from the original LLAGN sample exhibit very peculiar positions in the different plots. They are detected only very weakly at $\sim 3 \sigma_{\mathrm{BG}}$ and were excluded from the correlation and regression analysis because of evidence that their observed fluxes at multiple wavelengths are heavily dominated by nonAGN processes. These two objects are now discussed separately.

\subsubsection{NGC 3125}

The X-ray analysis of Chandra data published by Dudik et al. (2005) indicated an AGN in this object, while Zhang et al. (2009) reports a point-like X-ray nucleus embedded in soft emission based on the same data. On the other hand, this galaxy is known to harbor young and extreme Wolf-Rayet star clusters, two of which have been confirmed by optical and near-infrared observations (Hadfield \& Crowther 2006), one directly in its galactic nucleus. In addition, no broad optical emission lines have been detected and it is optically classified as starburst (Kewley et al. 2001). Therefore, it is unclear whether NGC 3125 really harbors an AGN, or if the X-ray emission originates from X-ray binaries or an ultra-luminous X-ray source (ULX) inside or near the star cluster. The MIR emission may either originate from dust heated by an AGN or by the central star cluster on which the VISIR imaging was centered. The latter is favored because this object is also the only spatially resolved one in the LLAGN sample. Note that the second and fainter star cluster at $\sim 10^{\prime \prime}$ to the east was not detected. The VISIR photometry and the IRS spectrum agree well in the continuum, indicating that the emission seen by IRS originates from a compact nucleus. On the other hand, the strong PAH emission feature in the IRS spectrum seems to be absent from the VISIR data. By assuming that the PAH $11.3 \mu \mathrm{m}$ emission is still present in the nucleus the maximum contribution of star-formation $c_{<}^{\mathrm{SB}}$ is only $\leq 51 \%$. However, complex molecules are probably also destroyed in close proximity of extremely massive young star forming regions. Evidence is strong that the Wolf-Rayet star cluster dominates the MIR emission of NGC 3125, which is the reason for its peculiar position with respect to the luminosity correlations.

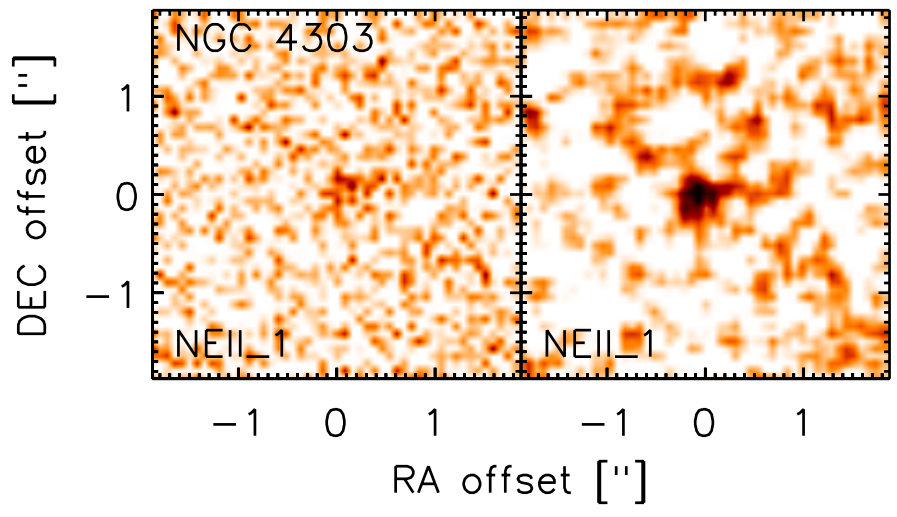

Fig. 7. VISIR image of NGC 4303 in NeIIref1. Left side: co-added image $(50 \times 50$ pixel $)$; right side: the same image but smoothed before co-adding; black color corresponds to $\langle\mathrm{BG}\rangle+5 \sigma_{\mathrm{BG}}$ with a linear scaling.

Hence, the observed MIR flux should be regarded as an upper limit for any dusty-torus emission of a putative AGN in NGC 3125.

\subsubsection{NGC $4303-M 61$}

This object was claimed to be undetected in Horst et al. (2008). A careful re-analysis of the NeIIref1 image revealed a very weak detection at a low $\sigma \sim 2$ (compare Fig. 7). Similar to NGC 1667, the negative beams were found at the expected positions. Furthermore, the detection significance increases in the smoothed co-added image. Still, the MIR values calculated for this object are unreliable owing to the very low $S / N$. Because the flux of this object is very low, it will unfortunately be very difficult to improve the $S / N$ with any additional observations. NGC 4303 was classified as having an HII nucleus by Ho et al. (1995), although the optical line ratios are close to the AGN regime. Its X-ray luminosity is determined by Tzanavaris \& Georgantopoulos (2007) also based on Chandra data. They conclude that this object is a good AGN candidate even with a low luminosity and without a detected $\mathrm{Fe} \mathrm{K} \alpha$ emission line. 
Jiménez-Bailón et al. (2003) derive a 0.3 dex lower value from the same data. Both works report a hard X-ray core surrounded by a softer emission environment possibly associated with a circum-nuclear starburst ring. Colina et al. (2002) find evidence for a young star cluster present in the nucleus. This could contribute significantly to or even dominate the MIR emission at these low luminosities. This would explain the object's extreme position in the luminosity plane far off from the correlation. Unfortunately, there is no IRS spectrum covering the $N$-band region. The nuclear star cluster might easily dominate the observed MIR emission similar to NGC 3125, which would then lead to the observed excess. Note that off-nuclear young clusters with comparable MIR luminosities are found for example in NGC 1365 (Galliano et al. 2008) and would be similarly unresolved at the distance of NGC 4303.

\section{Conclusions}

We presented VLT/VISIR mid-infrared ( $N$-band) imaging of 17 nearby LLAGN (and one NELG) at a spatial resolution of $\sim 0$ !. 35 in two narrow-band filters. The images show point-like sources in the seven detected LLAGN, and we found no extended emission nearby the nucleus.

Possible sources of this unresolved MIR emission are nuclear star-formation, the putative dusty torus, the accretion disk, the jet, or the narrow-line region. Any significant contribution to the $12 \mu \mathrm{m}$ continuum by the latter is considered unlikely (Groves et al. 2006; Mor et al. 2009). The other possibilities were investigated by three different methods:

Firstly, a comparison to the MIR-X-ray luminosity correlation found for brighter AGN: Using X-ray luminosities taken from the literature, all detections plus nine additional LLAGN from the archive were compared to the sample from $\mathrm{G}+09$. The MIR excess (or X-ray deficit) of the LLAGN is not statistically significant. Instead, the MIR-X-ray correlation from G+09 is also valid for the LLAGN, and it extends to luminosities of the order of of $<10^{41} \mathrm{erg} / \mathrm{s}$ with a small scatter. It is noteworthy that the local starburst galaxies with globally measured MIR and X-ray luminosities show a similar correlation with a comparable slope close to 1 but with $\sim 2.6$ orders of magnitude difference in normalization. The two peculiar outliers, NGC 3125 and NGC 4303, are explained by dominant nuclear star clusters.

Secondly, the comparison to IRAS and Spitzer/IRS showed that the IRAS photometry is dominated by the host galaxy in most cases, with decreasing AGN contribution with decreasing X-ray luminosities. The comparison to small-aperture IRS $N$-band spectra yields much better constraints on the nuclear star-formation. In particular, the PAH $11.3 \mu \mathrm{m}$ emission line flux is regarded as a star-formation indicator. A typical starburst template SED scaled by the line flux observed in IRS for the individual LLAGN constrains the maximum $12 \mu \mathrm{m}$ continuum contribution of a putative nuclear starburst. For two objects, NGC 1097 and NGC 1566, strong PAH emission is indicated even at the $0.4^{\prime \prime}$ scale. Star formation might dominate their nuclei in the MIR, but in general the weakness or absence of the PAH feature in $75 \%$ of the LLAGN restricts the nuclear non-AGN contamination to $\lesssim 30 \%$, often much less, and thus disfavors star-formation as the dominating MIR source in LLAGN.

Thirdly, for low accretion rates a change in the accretion structure of the AGN is proposed by several works, which should also affect the MIR properties of the LLAGN. However, the MIR-X-ray luminosity ratio is independent of the accretion rate, over at least 4 (possibly 6 ) orders of magnitude to $\lambda_{\text {Edd }} \sim 10^{-5}$.
These results do not prove the presence of a dusty torus, but they are consistent with its existence in all observed LLAGN. Consequently, the unification model might still hold for LLAGN, as already suggested by, e.g., Panessa et al. (2006) and Maoz (2007). Furthermore, Elitzur \& Ho (2009) recently determined a threshold for the disappearance of the torus at very low accretion rates depending on the bolometric luminosity. None of the detected LLAGN is below this threshold. On the other hand, if there were no torus and the MIR emission were dominated by the jet or ADAF, there would be a fortuitous agreement in both slope and normalization with the correlation for brighter (torus-dominated) Seyferts. In any case, that all AGN (Seyferts and LINERs) can be well-described by a single correlation without any strong offsets makes the MIR-X-ray correlation a very useful observational tool for converting between MIR and X-ray powers, irrespective of the nature of the individual AGN.

Increasing the sample size, in particular for the LINERs, would lead to much tighter constraints on the nature of the MIR emission in LLAGN. Unfortunately, current MIR facilities either lack the sensitivity or spatial resolution necessary to observe most LLAGN. On the other hand, additional investigations using additional multiwavelength data will yield more clues on the nature of the MIR emission and properties of LLAGN.

Acknowledgements. We thank the anonymous referee for many helpful suggestions that improved this work a lot. In addition, we also thank Sera Markoff for very valuable comments. This research made use of the NASA/IPAC Extragalactic Database (NED) which is operated by the Jet Propulsion Laboratory, California Institute of Technology, under contract with the National Aeronautics and Space Administration. We acknowledge the usage of the HyperLeda database (http://leda.univ-lyon1.fr). P.G. acknowledges a JAXA International Top Young Fellowship. This research is supported in part by a JSPS Grant in Aid number 21740152. S.H. acknowledges support by Deutsche Forschungsgemeinschaft (DFG) in the framework of a research fellowship ("Auslandsstipendium”). D.A. thanks Michael West for improving suggestions on the manuscript. He thanks Amelia Bayo for providing the IRS fluxes and the many motivating comments.

\section{References}

Akylas, A., \& Georgantopoulos, I. 2009, A\&A, 500, 999

Alonso-Herrero, A., Ramos Almeida, C., Mason, R., et al. 2011, ApJ, 736, 82 Antonucci, R. 1993, ARA\&A, 31, 473

Baes, M., Clemens, M., Xilouris, E. M., et al. 2010, A\&A, 518, L53 Bassani, L., Dadina, M., Maiolino, R., et al. 1999, ApJSS, 121, 473 Bettoni, D., Falomo, R., Fasano, G., \& Govoni, F. 2003, A\&A, 399, 869 Bianchi, S., Guainazzi, M., Matt, G., et al. 2005, A\&A, 442, 185

Bianchi, S., Bonilla, N. F., Guainazzi, M., Matt, G., \& Ponti, G. 2009, A\&A, 501,915

Blakeslee, J. P., Lucey, J. R., Barris, B. J., Hudson, M. J., \& Tonry, J. L. 2001, MNRAS, 327, 1004

Brandl, B. R., Bernard-Salas, J., Spoon, H. W. W., et al. 2006, ApJ, 653, 1129 Brightman, M., \& Nandra, K. 2011, MNRAS, 413, 1206

Burtscher, L., Jaffe, W., Raban, D., et al. 2009, ApJL, 705, 53

Cappellari, M., Verolme, E. K., van der Marel, R. P., et al. 2002, ApJ, 578, 787

Cappellari, M., Neumayer, N., Reunanen, J., et al. 2009, MNRAS, 394, 660

Cappi, M., Panessa, F., Bassani, L., et al. 2006, A\&A, 446, 459

Carrillo, R., Masegosa, J., Dultzin-Hacyan, D., \& Ordoñez, R. 1999, Rev. Mex. Astron. Astrofis., 35, 187

Cohen, M., Walker, R. G., Carter, B., et al. 1999, AJ, 117, 1864

Colina, L., Gonzalez Delgado, R., Mas-Hesse, J. M., \& Leitherer, C. 2002, ApJ, 579,545

Diamond-Stanic, A. M., \& Rieke, G. H. 2010, ApJ, 724, 140

Diamond-Stanic, A. M., \& Rieke, G. H. 2011, [arXiv: 1106. 3565]

Diamond-Stanic, A. M., Rieke, G. H., \& Rigby, J. R. 2009, ApJ, 698, 623

Dudik, R. P., Satyapal, S., Gliozzi, M., \& Sambruna, R. M. 2005, ApJ, 620, 113

Dudik, R. P., Satyapal, S., \& Marcu, D. 2009, ApJ, 691, 1501

Dullemond, C. P., \& van Bemmel, I. M. 2005, A\&A, 436, 47

Elitzur, M., \& Ho, L. C. 2009, ApJ, 701, L91

Elitzur, M., \& Shlosman, I. 2006, ApJ, 648, L101

Falcke, H., \& Markoff, S. 2000, A\&A, 362, 113

Ferrarese, L. \& Merritt, D. 2000, ApJ, 539, L9 
Ferrarese, L., Livio, M., Freedman, W., et al. 1996, ApJ, 468, L95 Ferrarese, L., Mould, J. R., Stetson, P. B., et al. 2007, ApJ, 654, 186 Galliano, E., Alloin, D., Pantin, E., Lagage, P. O., \& Marco, O. 2005, A\&A, 438, 803

Galliano, E., Alloin, D., Pantin, E., et al. 2008, A\&A, 492, 3

Gallimore, J. F., Yzaguirre, A., Jakoboski, J., et al. 2010, ApJS, 187, 172

Gandhi, P., Horst, H., Smette, A., et al. 2009, A\&A, 502, 457

Garcia-Rissmann, A., Vega, L. R., Asari, N. V., et al. 2005, MNRAS, 359, 765

Gebhardt, K., Bender, R., Bower, G., et al. 2000, ApJ, 539, L13

Gebhardt, K., Adams, J., Richstone, D., et al. 2011, ApJ, 729, 119

González-Martín, O., Masegosa, J., Márquez, I., \& Guainazzi, M. 2009a, ApJ, 704,1570

González-Martín, O., Masegosa, J., Márquez, I., Guainazzi, M., \& JiménezBailón, E. 2009b, A\&A, 506, 1107

Gorjian, V., Werner, M. W., Jarrett, T. H., Cole, D. M., \& Ressler, M. E. 2004, ApJ, 605, 156

Goulding, A. D., \& Alexander, D. M. 2009, MNRAS, 398, 1165

Grier, C. J., Mathur, S., Ghosh, H., \& Ferrarese, L. 2011, ApJ, 731, 60

Grossan, B. 2004 [arXiv: astro-ph/0405190]

Groves, B., Dopita, M., \& Sutherland, R. 2006, A\&A, 458, 405

Gültekin, K., Richstone, D. O., Gebhardt, K., et al. 2009, ApJ, 698, 198

Haas, M., Siebenmorgen, R., Schulz, B., Krügel, E., \& Chini, R. 2005, A\&A, 442, L39

Haas, M., Siebenmorgen, R., Pantin, E., et al. 2007, A\&A, 473, 369

Hadfield, L. J., \& Crowther, P. A. 2006, MNRAS, 368, 1822

Hardcastle, M. J., Evans, D. A., \& Croston, J. H. 2009, MNRAS, 396, 1929

Hernán-Caballero, A., \& Hatziminaoglou, E. 2011, MNRAS, 414, 500

Ho, L. C. 2008, ARA\&A, 46, 475

Ho, L. C. \& Ulvestad, J. S. 2001, ApJS, 133, 77

Ho, L. C., Filippenko, A. V., \& Sargent, W. L. 1995, ApJS, 98, 477

Ho, L. C., Feigelson, E. D., Townsley, L. K., et al. 2001, ApJ, 549, L51

Ho, L. C., Filippenko, A. V., \& Sargent, W. L. W. 1997, ApJS, 112, 315

Ho, L. C., Greene, J. E., Filippenko, A. V., \& Sargent, W. L. W. 2009, ApJSS, 183,1

Hönig, S. F. \& Beckert, T. 2007, MNRAS, 380, 1172

Hönig, S. F. \& Kishimoto, M. 2010, A\&A, 523, A27

Hönig, S. F., Beckert, T., Ohnaka, K., \& Weigelt, G. 2006, A\&A, 452, 459

Hönig, S. F., Kishimoto, M., Gandhi, P., et al. 2010, A\&A, 515, A23

Horst, H., Smette, A., Gandhi, P., \& Duschl, W. J. 2006, A\&A, 457, L17

Horst, H., Gandhi, P., Smette, A., \& Duschl, W. J. 2008, A\&A, 479, 389

Horst, H., Duschl, W. J., Gandhi, P., \& Smette, A. 2009, A\&A, 495, 137

Hudaverdi, M., Kunieda, H., Tanaka, T., et al. 2006, PASJ, 58, 931

Humphrey, P. J., \& Buote, D. A. 2006, ApJ, 639, 136

Isobe, T., Feigelson, E. D., Akritas, M. G., \& Babu, G. J. 1990, ApJ, 364, 104

Jacoby, G. H., Branch, D., Ciardullo, R., et al. 1992, PASP, 104, 599

Jaffe, W., Meisenheimer, K., Röttgering, H. J. A., et al. 2004, Nature, 429, 47

Jensen, J. B., Tonry, J. L., Barris, B. J., et al. 2003, ApJ, 583, 712

Jiménez-Bailón, E., Santos-Lleó, M., Mas-Hesse, J. M., et al. 2003, ApJ, 593, 127

Kelly, B. C. 2007, ApJ, 665, 1489

Kewley, L. J., Heisler, C. A., Dopita, M. A., \& Lumsden, S. 2001, ApJS, 132, 37 Kim, D., Barkhouse, W. A., Romero-Colmenero, E., et al. 2006, ApJ, 644, 829

Krabbe, A., Böker, T., \& Maiolino, R. 2001, ApJ, 557, 626

Lagage, P. O., Pel, J. W., Authier, M., et al. 2004, The Messenger, 117, 12

LaMassa, S. M., Heckman, T. M., Ptak, A., et al. 2010, ApJ, 720, 786

Leipski, C., Antonucci, R., Ogle, P., \& Whysong, D. 2009, ApJ, 701, 891

Levenson, N. A., Heckman, T. M., Krolik, J. H., Weaver, K. A., \& Życki, P. T. 2006, ApJ, 648, 111

Levenson, N. A., Radomski, J. T., Packham, C., et al. 2009, ApJ, 703, 390

Lewis, K. T., \& Eracleous, M. 2006, ApJ, 642, 711

Liu, J., \& Bregman, J. N. 2005, ApJS, 157, 59

Lobban, A. P., Reeves, J. N., Porquet, D., et al. 2011, MNRAS, 411, 2353

Lodato, G., \& Bertin, G. 2003, A\&A, 398, 517

Lutz, D., Maiolino, R., Spoon, H. W. W., \& Moorwood, A. F. M. 2004, A\&A, 418,465

Maccarone, T. J., Kundu, A., \& Zepf, S. E. 2003, ApJ, 586, 814

Madore, B. F., Freedman, W. L., Silbermann, N., et al. 1999, ApJ, 515, 29

Maoz, D. 2007, MNRAS, 377, 1696

Markoff, S., Nowak, M., Young, A., et al. 2008, ApJ, 681, 905

Mason, R. E., Levenson, N. A., Packham, C., et al. 2007, ApJ, 659, 241

McElroy, D. B. 1995, ApJSS, 100, 105

Mei, S., Blakeslee, J. P., Côté, P., et al. 2007, ApJ, 655, 144

Meléndez, M., Kraemer, S. B., Armentrout, B. K., et al. 2008, ApJ, 682, 94

Mor, R., Netzer, H., \& Elitzur, M. 2009, ApJ, 705, 298
Mullaney, J. R., Alexander, D. M., Goulding, A. D., \& Hickox, R. C. 2011, MNRAS, 414, 1082

Murphy, K. D., Yaqoob, T., \& Terashima, Y. 2007, ApJ, 666, 96

Nagar, N. M., Falcke, H., \& Wilson, A. S. 2005, A\&A, 435, 521

Narayan, R., \& Yi, I. 1994, ApJ, 428, L13

Nemmen, R. S., Storchi-Bergmann, T., Yuan, F., et al. 2006, ApJ, 643, 652

Nenkova, M., Ivezić, v., \& Elitzur, M. 2002, ApJ, 570, L9

Nenkova, M., Sirocky, M. M., Nikutta, R., Ivezić, v., \& Elitzur, M. 2008, ApJ, 685,160

Nicastro, F., Martocchia, A., \& Matt, G. 2003, ApJ, 589, L13

Panessa, F., Bassani, L., Cappi, M., et al. 2006, A\&A, 455, 173

Papadakis, I. E., Ioannou, Z., Brinkmann, W., \& Xilouris, E. M. 2008, A\&A 490, 995

Pappa, A., Georgantopoulos, I., Stewart, G. C., \& Zezas, A. L. 2001, MNRAS, 326, 995

Paturel, G., Petit, C., Prugniel, P., et al. 2003, A\&A, 412, 45

Perlman, E. S., Mason, R. E., Packham, C., et al. 2007, ApJ, 663, 808

Peterson, B. M., Ferrarese, L., Gilbert, K. M., et al. 2004, ApJ, 613, 682

Press, W. H., Teukolsky, S. A., Vetterling, W. T., \& Flannery, B. P. 1992, Numerical recipes in FORTRAN. The art of scientific computing

Raban, D., Jaffe, W., Röttgering, H., Meisenheimer, K., \& Tristram, K. R. W. 2009, MNRAS, 394, 1325

Ramos Almeida, C., Levenson, N. A., Rodríguez Espinosa, J. M., et al. 2009, ApJ, 702, 1127

Ranalli, P., Comastri, A., \& Setti, G. 2003, A\&A, 399, 39

Reunanen, J., Prieto, M. A., \& Siebenmorgen, R. 2010, MNRAS, 402, 879

Risaliti, G., Elvis, M., Fabbiano, G., et al. 2007, ApJ, 659, L111

Sanders, D. B., Mazzarella, J. M., Kim, D., Surace, J. A., \& Soifer, B. T. 2003, AJ, 126, 1607

Sansom, A. E., O'Sullivan, E., Forbes, D. A., Proctor, R. N., \& Davis, D. S. 2006, MNRAS, 370, 1541

Satyapal, S., Sambruna, R. M., \& Dudik, R. P. 2004, A\&A, 414, 825

Satyapal, S., Dudik, R. P., O'Halloran, B., \& Gliozzi, M. 2005, ApJ, 633, 86

Schartmann, M., Meisenheimer, K., Camenzind, M., et al. 2008, A\&A, 482, 67

Schwope, A., Hasinger, G., Lehmann, I., et al. 2000, Astron. Nachr., 321, 1

Shakura, N. I., \& Sunyaev, R. A. 1973, A\&A, 24, 337

Shi, Y., Rieke, G. H., Smith, P., et al. 2010, ApJ, 714, 115

Shu, X. W., Liu, T., \& Wang, J. X. 2010a, ApJ, 722, 96

Shu, X. W., Yaqoob, T., \& Wang, J. X. 2010b, ApJS, 187, 581

Siebenmorgen, R., Haas, M., Pantin, E., et al. 2008, A\&A, 488, 83

Slee, O. B., Sadler, E. M., Reynolds, J. E., \& Ekers, R. D. 1994, MNRAS, 269, 928

Snijders, L., van der Werf, P. P., Brandl, B. R., et al. 2006, ApJ, 648, L25

Springob, C. M., Masters, K. L., Haynes, M. P., Giovanelli, R., \& Marinoni, C. 2007, ApJS, 172, 599

Starling, R. L. C., Page, M. J., Branduardi-Raymont, G., et al. 2005, MNRAS, 356,727

Storchi-Bergmann, T., Nemmen, R. S., Spinelli, P. F., et al. 2005, ApJ, 624, L13

Terashima, Y., Ho, L. C., \& Ptak, A. F. 2000, ApJ, 539, 161

Tremaine, S., Gebhardt, K., Bender, R., et al. 2002, ApJ, 574, 740

Tristram, K. R. W., \& Schartmann, M. 2011, A\&A, 531, A99

Tristram, K. R. W., Raban, D., Meisenheimer, K., et al. 2009, A\&A, 502, 67

Tully, R. B. 1988, Nearby galaxies catalog (Cambridge, New York: Cambridge University Press)

Tzanavaris, P., \& Georgantopoulos, I. 2007, A\&A, 468, 129

van der Wolk, G., Barthel, P. D., Peletier, R. F., \& Pel, J. W. 2010, A\&A, 511, A64

Vasudevan, R. V., \& Fabian, A. C. 2007, MNRAS, 381, 1235

Vasudevan, R. V., Mushotzky, R. F., Winter, L. M., \& Fabian, A. C. 2009, MNRAS, 399, 1553

Vasudevan, R. V., Fabian, A. C., Gandhi, P., Winter, L. M., \& Mushotzky, R. F. 2010, MNRAS, 402, 1081

Véron-Cetty, M., \& Véron, P. 2010, A\&A, 518, A10

Wang, J., \& Zhang, E. 2007, ApJ, 660, 1072

Willick, J. A., Courteau, S., Faber, S. M., et al. 1997, ApJS, 109, 333

Winter, L. M., Lewis, K. T., Koss, M., et al. 2010, ApJ, 710, 503

Woo, J., \& Urry, C. M. 2002, ApJ, 579, 530

Woo, J., Treu, T., Barth, A. J., et al. 2010, ApJ, 716, 269

Yuan, F. 2007, in The Central Engine of Active Galactic Nuclei, ASP Conf. Ser., 373, 95

Yuan, F., \& Cui, W. 2005, ApJ, 629, 408

Yuan, F., Yu, Z., \& Ho, L. C. 2009, ApJ, 703, 1034

Zhang, W. M., Soria, R., Zhang, S. N., Swartz, D. A., \& Liu, J. F. 2009, ApJ, 699,281 


\section{Appendix A: Brighter AGN properties}

For completeness we state the relevant properties of the AGN sample from G+09 in Table A.1.

Table A.1. Luminosities and other properties for the G+09 minus LLAGN.

\begin{tabular}{|c|c|c|c|c|c|c|}
\hline Object & Seyfert type & $\begin{array}{c}\log L_{2-10 \mathrm{keV}} \\
{[\mathrm{erg} / \mathrm{s}]}\end{array}$ & $\begin{array}{c}\log \lambda L_{12.3 \mu \mathrm{m}} \\
{[\mathrm{erg} / \mathrm{s}]}\end{array}$ & $\begin{array}{c}\log M_{\mathrm{BH}} \\
{\left[M_{\odot}\right]}\end{array}$ & Ref. & $\log \lambda$ \\
\hline Fairall9 & 1.2 & $43.87 \pm 0.15$ & $44.51 \pm 0.03$ & 7.91 & 8 & -1.0 \\
\hline NGC 526A & 1.9 & $43.14 \pm 0.10$ & $43.70 \pm 0.08$ & 8.10 & 2 & -1.9 \\
\hline SWIFT J0138.6-4001 & 2 & $43.59 \pm 0.30$ & $43.10 \pm 0.04$ & 9.68 & 9 & -3.6 \\
\hline Mrk 590 & 1 & $43.61 \pm 0.25$ & $43.55 \pm 0.06$ & 7.66 & 10 & -1.3 \\
\hline NGC 1068 & $1 \mathrm{~h}$ & $43.40 \pm 0.30$ & $43.80 \pm 0.03$ & 6.93 & 4 & -0.6 \\
\hline ESO 209-G012 & 1.5 & $43.65 \pm 0.15$ & $44.22 \pm 0.06$ & & & \\
\hline NGC 3081 & $1 \mathrm{~h}$ & $42.60 \pm 0.30$ & $42.64 \pm 0.04$ & 7.31 & 2 & -1.9 \\
\hline ESO 263-G013 & 2 & $43.30 \pm 0.40$ & $43.42 \pm 0.02$ & & $\ldots$ & $\ldots$ \\
\hline NGC 3281 & 2 & $43.30 \pm 0.15$ & $43.76 \pm 0.02$ & 7.72 & 2 & -1.4 \\
\hline NGC 3393 & 2 & $43.04 \pm 0.30$ & $42.90 \pm 0.02$ & 8.09 & 2 & -2.4 \\
\hline NGC 3783 & 1.5 & $43.21 \pm 0.15$ & $43.52 \pm 0.05$ & 7.45 & 10 & -1.3 \\
\hline NGC 4388 & $1 \mathrm{~h}$ & $42.24 \pm 0.30$ & $42.45 \pm 0.03$ & 7.10 & 3 & -2.0 \\
\hline NGC 4507 & $1 \mathrm{~h}$ & $43.30 \pm 0.15$ & $43.67 \pm 0.04$ & 8.39 & 9 & -2.2 \\
\hline NGC 4593 & 1 & $42.93 \pm 0.20$ & $43.18 \pm 0.08$ & 6.97 & 10 & -1.2 \\
\hline IC 3639 & $1 \mathrm{~h}$ & $43.62 \pm 0.50$ & $43.51 \pm 0.02$ & 6.75 & 2 & -0.4 \\
\hline ESO 323-G032 & 1.9 & $42.43 \pm 0.40$ & $42.96 \pm 0.04$ & & 2 & $\ldots$ \\
\hline NGC 4992 & 2 & $43.23 \pm 0.30$ & $43.36 \pm 0.02$ & 8.56 & 9 & -2.5 \\
\hline IRAS 13197-1627 & $1 \mathrm{~h}$ & $42.78 \pm 0.20$ & $44.07 \pm 0.03$ & 8.28 & 9 & -2.1 \\
\hline NGC 5135 & 2 & $43.00 \pm 0.50$ & $43.06 \pm 0.05$ & 7.30 & 2 & -1.5 \\
\hline MGC-06-30-15 & 1.5 & $42.74 \pm 0.20$ & $43.07 \pm 0.06$ & 6.73 & 2 & -1.1 \\
\hline NGC 5728 & 1.9 & $42.80 \pm 0.30$ & $42.69 \pm 0.03$ & 8.20 & 5 & -2.7 \\
\hline NGC 5995 & 2 & $43.54 \pm 0.15$ & $44.11 \pm 0.06$ & 7.11 & 7 & -0.5 \\
\hline ESO 138-G001 & 2 & $43.00 \pm 0.30$ & $43.54 \pm 0.02$ & 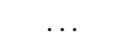 & $\cdots$ & $\ldots$ \\
\hline NGC 6300 & 2 & $42.30 \pm 0.30$ & $42.69 \pm 0.02$ & 6.77 & 2 & -1.5 \\
\hline ESO 103-G035 & 2 & $43.44 \pm 0.15$ & $43.74 \pm 0.03$ & 7.73 & 2 & -1.4 \\
\hline ESO 141-G055 & 1 & $43.90 \pm 0.15$ & $44.04 \pm 0.11$ & 7.91 & 7 & -1.2 \\
\hline NGC 6814 & 1.5 & $42.12 \pm 0.40$ & $42.07 \pm 0.02$ & 7.28 & 8 & -2.4 \\
\hline Mrk 509 & 1.5 & $44.10 \pm 0.15$ & $44.18 \pm 0.07$ & 7.86 & 8 & -1.0 \\
\hline PKS 2048-57 & $1 \mathrm{~h}$ & $42.84 \pm 0.20$ & $43.82 \pm 0.05$ & 7.74 & 8 & -1.7 \\
\hline PG 2130+099 & 1.5 & $43.65 \pm 0.20$ & $44.53 \pm 0.07$ & 7.91 & 6 & -1.1 \\
\hline NGC 7172 & 2 & $42.76 \pm 0.40$ & $42.79 \pm 0.07$ & 7.64 & 2 & -2.1 \\
\hline $3 \mathrm{C} 445$ & 1.5 & $44.26 \pm 0.15$ & $44.50 \pm 0.06$ & 8.15 & 2 & -1.0 \\
\hline NGC 7314 & $1 \mathrm{~h}$ & $42.20 \pm 0.15$ & $41.96 \pm 0.12$ & 7.84 & 9 & -3.0 \\
\hline NGC 7469 & 1.5 & $43.15 \pm 0.10$ & $43.92 \pm 0.03$ & 7.06 & 10 & -0.8 \\
\hline NGC 7674 & $1 \mathrm{~h}$ & $44.56 \pm 0.50$ & $44.31 \pm 0.03$ & 7.59 & 3 & -0.4 \\
\hline NGC 7679 & 2 & $42.52 \pm 0.15$ & $42.82 \pm 0.15$ & 6.35 & 3 & -0.9 \\
\hline
\end{tabular}

Notes. References for the black hole masses. (1) Bettoni et al. (2003); (2) Garcia-Rissmann et al. (2005); (3) Hyperleda database (Paturel et al. 2003); (4) Lodato \& Bertin (2003); (5) Lewis \& Eracleous (2006); (6) McElroy (1995); (7) Peterson et al. (2004); (8) Wang \& Zhang (2007); (9) Woo \& Urry (2002); (10) Winter et al. (2010); (11) Woo et al. (2010). $\log \lambda=\log \left(L_{\mathrm{Bol}} / L_{\mathrm{Edd}}\right)$ is the Eddington ratio. 


\section{Appendix B: Additional notes on individual objects}

\section{B.1. IC 1459}

Detailed X-ray modeling of Chandra observations of this object are presented in González-Martín et al. (2009b). Satyapal et al. (2004) give comparable model parameters for the same data set. For the X-ray luminosity the mean of both works was used. There is no [OIII] $\lambda 5007 \AA$ or [OIV] $\lambda 25.89 \mu \mathrm{m}$ luminosity measurement in the literature. The $\mathrm{X}$-ray data suggest that the object is not Compton-thick. In addition, a compact radio core was detected by Slee et al. (1994), confirming the AGN nature of this object. Accordingly, the non-detection with VISIR may be explainable by the bad weather conditions during the observations of IC 1459.

\section{B.2. NGC 676}

The only redshift-independent distance measurement for NGC 676 available in the literature is by Tully (1988) employing the Tully-Fisher relation, and this is used in this work. This object is included in the Palomar optical spectroscopic survey (Ho et al. 1995). But it was not detected in radio by Ho \& Ulvestad (2001). The used X-ray data based on XMM-Newton is published in Panessa et al. (2006). They interpret this object as Comptonthick in accordance with Akylas \& Georgantopoulos (2009) based on the same data. This is also supported by the relatively strong [OIII] emission (Panessa et al. 2006). Unfortunately, there are no Spitzer data available, and therefore no [OIV] measurement.

\section{B.3. NGC 1052}

The X-ray properties of NGC 1052 are described in GonzálezMartín et al. (2009b) based on Chandra data. The derived X-ray luminosity is very similar to the one given in Satyapal et al. (2004) for the same observation after correction for the different distances. The mean of both was used in this work. Counting as a prototypical LINER, NGC 1052 is surprisingly bright and obscured in X-rays. While the [OIII] luminosity agrees with the X-ray luminosity (González-Martín et al. 2009a), no [OIV] emission line has been detected (Satyapal et al. 2004). Very recently Brightman \& Nandra (2011) published high S /N XMMNewton data of this object, stating a higher intrinsic $L_{2-10 \mathrm{keV}}=$ $10^{41.5} \mathrm{erg} / \mathrm{s}$, which would bring this object closer to the correlation.

\section{B.4. NGC 1097}

The X-ray luminosity is taken from Nemmen et al. (2006) using Chandra observations. It is interesting to note that the measured [OIV] brightness would imply a much higher X-ray luminosity $\left(L_{\mathrm{X}} \sim 10^{42.2} \mathrm{erg} / \mathrm{s}\right)$ according to the correlation found between both (Diamond-Stanic et al. 2009), while the [OIII] luminosity implies a lower value $\left(L_{\mathrm{X}} \sim 10^{40.0} \mathrm{erg} / \mathrm{s}\right)$ using the [OIII]:X-ray correlation (Panessa et al. 2006). At a larger scale $\left(\sim 10^{\prime \prime}\right)$ the well-known starburst ring is evident in both filters but is only partly visible in the small VISIR field of view. The infrared properties of the nucleus have already been investigated in detail by Mason et al. (2007). They find the absence of PAH $3.3 \mu \mathrm{m}$ emission at subarcsec scale, which seems to be typical for strong radiation fields. On the other hand, attempts to fit the infrared data with torus models failed, which led them to the conclusion that the torus is absent or weak. Instead, a nuclear star-forming region, co-existing with the LLAGN, is assumed to dominate the observed MIR emission. The likely PAH $11.3 \mu \mathrm{m}$ emission in the VISIR data indicates that the nucleus is indeed dominated by emission coming from such a star-forming region. This also matches the finding of Storchi-Bergmann et al. (2005) of UV evidence for a nuclear star cluster inside the innermost 9 parsec of NGC 1097. But on the other hand, the MIR excess relative to the luminosity correlation is consistent with the co-existence of star-forming region and a torus inside the nucleus of NGC 1097. To distinguish these components better, additional MIR observations are needed.

\section{B.5. NGC 1386}

NGC 1386 is one of the objects for which the different methods used to determine the distance show the largest discrepancies ( $\sim 50 \%$ variation). Because this object is very likely Comptonthick it is difficult to derive a reliable X-ray luminosity. We followed the approach of Levenson et al. (2006) and estimated the luminosity by using the [OIII] line strength. The derived value is consistent with the $\mathrm{Fe} \mathrm{K} \alpha$ emission line properties observed by Chandra that are analyzed in the same work. It also agrees with the [OIV] brightness stated in Diamond-Stanic et al. (2009) and LaMassa et al. (2010). Therefore, all available evidence favors a Compton-thick nature for this object.

\section{B.6. NGC 1404}

This elliptical galaxy is falling into the center of the Fornax cluster, which leads to large differences in the different distance measurement methods. It is classified as NELG in the optical. No evidence for AGN activity was found in the literature. Nevertheless, it is relatively bright in X-rays with a luminosity of $L_{X} \sim 10^{41} \mathrm{erg} / \mathrm{s}$ reported by Kim et al. (2006) based on Chandra observations. Because this value was calculated for the whole galaxy including soft X-ray emission, it has to be regarded as an upper limit for any Compton-thin AGN in this object. Very recently, Grier et al. (2011) re-examined the Chandra data and found a clear detection of a nuclear point source with $L_{\mathrm{X}} \sim 10^{40} \mathrm{erg} / \mathrm{s}$, which makes a LLAGN in this object likely. However, we treated NGC 1404 as a non-AGN galaxy and comparison object only.

\section{B.7. NGC 1566}

Similar to NGC 1386, NGC 1566 shows huge discrepancies in the different distance measurement methods. The only published X-ray data of this objects are from ROSAT (Liu \& Bregman 2005), where the emphasis was on off-nuclear ultra-luminous $X$ ray sources. The nuclear point-source was fitted only by a simple power-law. In the lack of any other available data, we still used these values. This X-ray luminosity agrees well with the predicted values from the [OIII] and [OIV] emission line measurements stated in Diamond-Stanic et al. (2009).

\section{B.8. NGC 1667}

Bianchi et al. (2005) found an X-ray flux variation of a factor of 100 for this object and interpreted the data in a way that the object is Compton-thick. Unfortunately, they give no intrinsic $\mathrm{X}$-ray luminosity or flux derived from the used XMM-Newton data. Therefore we used the value of Panessa et al. (2006) based on ASCA observations. These authors also interpreted the data 
in favor of Compton-thickness. This is supported by the appearance of a strong $\mathrm{Fe} \mathrm{K} \alpha$ line (Bassani et al. 1999). On the other hand, the same ASCA data were originally used by Pappa et al. (2001) for a Compton-thin fit, giving an X-ray luminosity that is two orders of magnitude lower for a similar observed flux. The stated [OIII] measurements in Panessa et al. (2006) support a Compton-thick nature. The [OIV] emission (Diamond-Stanic et al. 2009; LaMassa et al. 2010) also predicted an intrinsic X-ray luminosity similar to the one for the Compton-thick case. The VISIR observations support this scenario because NGC 1667 is much closer to the MIR-X-ray correlation than in the unabsorbed scenario.

\section{B.9. NGC 3312}

NGC 3312 was classified as a LINER by Carrillo et al. (1999). The X-ray data were taken from Hudaverdi et al. (2006), who describe XMM-Newton observations of this object. No other X-ray data or Spitzer or [OIII] observations are available.

\section{B.10. NGC 4235}

We used the XMM-Newton data that was analyzed by Papadakis et al. (2008) and Bianchi et al. (2009) and yielded similar results. Panessa et al. (2006) derived a luminosity 0.6 dex higher but based on ASCA observations, which we treated with a lower priority because of their poorer spatial resolution. It is noteworthy that the properties of this type 1 LLAGN agree well with those of brighter objects, and it is therefore likely that NGC 4235 accretes in radiatively efficient mode as well (Papadakis et al. 2008).

\section{B.11. NGC $4261-3 C 270$}

González-Martín et al. (2009b) and Satyapal et al. (2005) arrived at very similar X-ray luminosities based on the same Chandra observation. It is interesting to note that van der Wolk et al. (2010) reports a non-detection with VISIR in the SiC filter for this source. We re-analyzed these observations and found a weak but clear detection. The measured flux matches the new observations presented here, and is six times higher than their reported upper limit ( $2 \mathrm{mJy})$. Additional evidence for MIR emission of hot dust in the nucleus of this galaxy has been found by Leipski et al. (2009). Therefore a dusty torus is likely present in NGC 4261, which agrees with the fact that the object aligns well with the MIR-X-ray correlation.

\section{B.12. NGC 4472}

This object was included in Horst et al. (2008) erroneously because the nuclear X-ray luminosity was ambiguous owing to many off-nuclear X-ray sources. Only an upper limit for the AGN $\left(L_{\mathrm{X}}<10^{39.32} \mathrm{erg} / \mathrm{s}\right)$ derived from Chandra observations is given in Panessa et al. (2006). On the other hand, in a very detailed analysis Maccarone et al. (2003) found a nuclear X-ray source with $L_{X} \sim 10^{39} \mathrm{erg} / \mathrm{s}$ in the same data. This agrees very well with the [OIII] emission line brightness stated in Diamond-Stanic et al. (2009) while the [OIV] data indicate a much higher luminosity $\left(L_{X} \sim 10^{41.4} \mathrm{erg} / \mathrm{s}\right)$. This seems to be the only indication of Compton-thickness, which is also not supported by the non-detection with VISIR. NGC 4472 might be a so-called "true" Seyfert 2 candidate - meaning that the broad emission lines are intrinsically absent rather than obscured by a torus (Nicastro et al. 2003). This was discussed in Akylas \& Georgantopoulos (2009), but the XMM-Newton data presented there are heavily contaminated by off-nuclear emission and therefore are unreliable. Unfortunately, we can reach no conclusion about the existence of a torus in NGC 4472.

\section{B.13. NGC $4486-M 87$}

We used the mean of the X-ray luminosities of González-Martín et al. (2009b) and Satyapal et al. (2004). Both were using the same Chandra data and state very comparable results. The flux visible in the Spitzer IRS spectrum matches the VISIR data well. Interestingly, Perlman et al. (2007) explained all observed MIR emission with synchrotron emission by the jet and conclude that there is probably no torus present in NGC 4486. Very recent Herschel/PACS and SPIRE observations (Baes et al. 2010) showed the absence of significant thermal components in the MIR to far-infrared SED on large-scale $\left(>10^{\prime \prime}\right)$ : a single power law can roughly fit the overall SED from MIR all way to the radio with a slope of $\sim-0.7$. But because of the comparably low spatial resolution no conclusion about the presence of a torus can be drawn from these data.

\section{B.14. NGC $4594-M 104$}

The X-ray properties are taken from González-Martín et al. (2009b) and Ho et al. (2001) based on Chandra with marginally different results. This object was detected in the $N$-band by Grossan (2004) with the Keck LWS. The reported nuclear flux at $10.2 \mu \mathrm{m}(11.1 \mathrm{mJy})$ is consistent with the upper limit we derived and would place this object close to the correlation. The Spitzer/IRS spectrum is very noisy and the much higher flux implies heavy non-nuclear contamination at this lower spatial resolution. Similar to NGC 4472, NGC 4594 is a so-called "true" Sy 2 candidate as well and was investigated in detail in Shi et al. (2010). They found strong silicate emission, indicating that this object possesses a face-on dusty torus. The combined multiwavelength properties, including X-ray and optical/UV imply that NGC 4594 indeed lacks broad emission lines, which contradicts the simple unification models.

\section{B.15. NGC 4698}

Cappi et al. (2006) and González-Martín et al. (2009b) give X-ray spectral fits for NGC 4698 with low absorption differing by 0.4 dex derived from XMM-Newton and Chandra data $\left(L_{X}=10^{39.39} \mathrm{erg} / \mathrm{s}\right.$ and $\left.10^{38.97} \mathrm{erg} / \mathrm{s}\right)$, respectively. But González-Martín et al. (2009a) interpreted NGC 4698 as Compton-thick using the same Chandra data. This conclusion was based on the [OIII] to X-ray flux ratio. Similarly high [OIII] fluxes were stated in Panessa et al. (2006) and Diamond-Stanic et al. (2009). In addition, a high X-ray obscuration is consistent with the [OIV] emission line brightness of the latter work. Therefore, the object is very likely Compton-thick, but with a large luminosity uncertainty. However, the MIR upper limit of NGC 4698 is consistent with both the Compton-thin or -thick scenario.

\section{B.16. NGC 5363}

Initial X-ray studies by González-Martín et al. (2009b) and Sansom et al. (2006) applied unobscured spectral fits to the available XMM-Newton observations and calculated a low X-ray 
luminosity $\left(L_{\mathrm{X}}=10^{39.68} \mathrm{erg} / \mathrm{s}\right.$ and $\left.10^{40.30} \mathrm{erg} / \mathrm{s}\right)$ for NGC 5363 . However, in the most recent analysis González-Martín et al. (2009a) give a much higher luminosity from the same data but with a Compton-thick model. This is supported by the [OIII] brightness and a relatively flat spectrum above $2 \mathrm{keV}$. Unfortunately, there is no Spitzer/IRS spectrum and no available [OIV] measurement. Consequently we adopted the Comptonthick fit.

\section{B.17. NGC 5813}

We used the X-ray luminosity of González-Martín et al. (2009b) based on Chandra for NGC 8813. The X-ray image shows extremely diffuse soft emission. But no hard emission core is visible, which makes an AGN in this object highly unlikely. On the other hand, it could be a Compton-thick object as argued in González-Martín et al. (2009a). But the [OIII] luminosity given in the same work predicts a only slightly higher X-ray luminosity than in the Compton-thin fit. The AGN nature is supported by the detection of a compact radio core by Nagar et al. (2005). But even if the Compton-thick luminosity $\left(L_{X}=10^{40.55} \mathrm{erg} / \mathrm{s}\right)$ is adopted, the non-detection with VISIR is not surprising and the derived upper limit does not constrain the correlation.

\section{B.18. NGC 7213}

The X-ray measurements by XMM-Newton (e.g., Starling et al. 2005), Chandra (Shu et al. 2010b) and Suzaku (Lobban et al. 2011) available in the literature give very similar X-ray luminosities for NGC 7213. We used the mean value. Because these come from different satellites and epochs, it seems unlikely that this object varies strongly in the X-rays. Therefore we adopted a smaller uncertainty for this object $(0.1 \mathrm{dex})$.

\section{B.19. NGC 7590}

XMM-Newton data revealed that the X-ray emission of NGC 7590 is dominated by an off-nuclear source and extended soft emission from the host galaxy (Shu et al. 2010a). Both dominate the older ASCA observation by Bassani et al. (1999), which has a poorer spatial resolution. Unfortunately, there are no Chandra observations available yet for this object. We used the estimated nuclear hard X-ray luminosity of Shu et al. (2010a). But note that based on the [OIII] to $2-10 \mathrm{keV}$ flux ratio, the object is likely Compton-thick, which would imply a much higher intrinsic value. This contradicts the hypothesis that this object is a "true" Sy 2 candidate (compare also Shi et al. 2010). The upper MIR flux limit derived from the VISIR observation does not help to constrain its nature because even an intrinsic $2-10 \mathrm{keV}$ luminosity higher by 2 orders of magnitude would be consistent with the correlation found. Similarly, the IRS spectrum is too noisy for a more detailed analysis and it remains unclear if the nucleus was detected at all.

\section{B.20. NGC 7626}

The Chandra observation of NGC 7626 has only been published by Humphrey \& Buote (2006) for the whole galaxy in the $0.1-10 \mathrm{keV}$ band. The [OIII] flux measured by Ho et al. (1997) predicts a much lower value $\left(L_{X}=10^{39.56} \mathrm{erg} / \mathrm{s}\right)$ and will be used in this study. Dudik et al. (2009) analyzed the forbidden emission lines visible in Spitzer data and concluded that this object might not be an AGN based on the non-detection of [NeV] and broad $\mathrm{H} \alpha$ emission. But the upper limit for the predicted Xray luminosity $\left(L_{X}<10^{41.03} \mathrm{erg} / \mathrm{s}\right)$ from the upper-limit of the [OIV] non-detection is consistent with the [OIII] prediction. In addition, a point source was detected in radio (Nagar et al. 2005). Accordingly, the nuclear nature of NGC 7626 remains uncertain. The non-detection with VISIR favors a low X-ray luminosity but the upper-limit would be consistent with the MIR-X-ray correlation in any case.

\section{B.21. NGC 7743}

The properties of this object are very similar to NGC 5363: it could either be highly absorbed or not absorbed at all: based on XMM-Newton González-Martín et al. (2009b) give an unobscured fit $\left(L_{X}=10^{39.5} \mathrm{erg} / \mathrm{s}\right)$, while Panessa et al. (2006), González-Martín et al. (2009a), and Akylas \& Georgantopoulos (2009) interpreted the X-ray data as Compton-thick. The [OIII] data (e.g., González-Martín et al. 2009a; Panessa et al. 2006) favor Compton-thickness in agreement with [OIV] measurements stated in Diamond-Stanic et al. (2009). Although there is no broad $\mathrm{H} \alpha$ line detected (Terashima et al. 2000), a compact radio core is present (Ho \& Ulvestad 2001). In summary, the true nature of this source remains unclear but it is likely Compton-thick. Therefore we used the X-ray luminosity given by GonzálezMartín et al. (2009b). Note however that a Compton-thin nature would agree better with the MIR-X-ray correlation. 